\title{
Long-term evolution and stability of Saturnian small satellites: Aegaeon, Methone, Anthe and Pallene
}

\author{
M. A. Munõz-Gutiérrez ${ }^{\star}$ and S. Giuliatti Winter \\ Universidade Estadual Paulista - UNESP, Grupo de Dinâmica Orbital e Planetologia, Av. Ariberto Pereira da Cunha, 333, Guaratinguetá-SP, \\ 12516-410, Brazil
}

Accepted 2017 June 16. Received 2017 June 6; in original form 2016 December 12

\begin{abstract}
Aegaeon, Methone, Anthe and Pallene are four Saturnian small moons, discovered by the Cassini spacecraft. Although their orbital characterization has been carried on by a number of authors, their long-term evolution has not been studied in detail so far. In this work, we numerically explore the long-term evolution, up to $10^{5} \mathrm{yr}$, of the small moons in a system formed by an oblate Saturn and the five largest moons close to the region: Janus, Epimetheus, Mimas, Enceladus and Tethys. By using frequency analysis, we determined the stability of the small moons and characterize, through diffusion maps, the dynamical behaviour of a wide region of geometric phase space, $a$ versus $e$, surrounding them. Those maps could shed light on the possible initial number of small bodies close to Mimas, and help to better understand the dynamical origin of the small satellites. We found that the four small moons are long-term stable and no mark of chaos is found for them. Aegaeon, Methone and Anthe could remain unaltered for at least $\sim 0.5 \mathrm{Myr}$, given the current configuration of the system. They remain well trapped in the corotation eccentricity resonances with Mimas in which they currently librate. However, perturbations from nearby resonances, such as Lindblad eccentricity resonances with Mimas, seem responsible for largest variations observed for Methone and Anthe. Pallene remains in a non-resonant orbit and it is the more stable, at least for $64 \mathrm{Myr}$. Nonetheless, it is affected by a quasi-resonance with Mimas, which induces long-term orbital oscillations of its eccentricity and inclination.
\end{abstract}

Key words: methods: numerical - planets and satellites: dynamical evolution and stability planets and satellites: rings.

\section{INTRODUCTION}

The Cassini space mission has been a highly successful endeavour that helped to expand significantly our knowledge and understanding of Saturn and its environment, both physically and dynamically. Amongst other important results, several new moons around Saturn were discovered thanks to images taken by the spacecraft. Four of such small moons, now called Aegaeon, Methone, Anthe and Pallene, lie between the position of the coorbital duet formed by Janus and Epimetheus, at $\sim 152000 \mathrm{~km}$ from Saturn centre, and roughly the inner edge of the E ring, where the moon Enceladus orbits, at $\sim 238000 \mathrm{~km}$ from Saturn centre. Those small bodies remain a challenge to be successfully characterized due to the complex dynamical environment they inhabit, nonetheless, such dynamical environment has started to be explored. In this work, we review our current knowledge of the four small moons and explore their

\footnotetext{
^E-mail: mmunoz.astro@gmail.com
}

dynamical environment and evolution on a wider and longer basis than current studies did.

Aegaeon was discovered and reported by Hedman et al. (2010). It is a small moon of roughly $0.33 \mathrm{~km}$ in diameter (Thomas et al. 2013) that lies in the middle of a prominent arc structure, located inside and close to the inner edge of the $\mathrm{G}$ ring. The $\mathrm{G}$ ring is a faint and thin ring formed by $\mu \mathrm{m}$ - to $\mathrm{cm}$-sized particles (Hedman et al. 2007), which extends from $\sim 165000$ to $\sim 175000 \mathrm{~km}$ from Saturn centre (Horányi et al. 2009). Some particles forming the G ring presumably emanate from collisions of micrometeoroids with the largest objects of the region; like Aegaeon itself, however, nowadays the origin of the ring remains uncertain. As already mentioned, the G ring possesses a unique bright arc located close to its inner edge, at $\sim 167500 \mathrm{~km}$ from Saturn centre, which covers $60^{\circ}$ in longitudinal extend, while has just $\sim 250 \mathrm{~km}$ of radial width. The arc is located at the 7:6 corotation eccentricity resonance (CER) with Mimas. It has been assumed that this resonance confines the longitudinal extent of the arc (Hedman et al. 2009).

Being the most prominent member of the arc of the $\mathrm{G}$ ring, Aegaeon orbits trapped at the 7:6 CER with Mimas, with a resonant 
argument given by $\varphi_{\mathrm{CER}}=7 \lambda_{\text {Mimas }}-6 \lambda_{\text {Aegaeon }}-\bar{\omega}_{\text {Mimas }}$, where $\lambda$ and $\bar{\omega}$ are the mean longitude and longitude of pericentre, respectively. This resonant argument librates with amplitude $\sim 10^{\circ}$ around $\sim 180^{\circ}$. However, Aegaeon's longitude deviates from the expected position, if one only considers its $\sim 10^{\circ}$ libration due to the CER. This small deviation has a period of $\sim 70 \mathrm{yr}$, similar to the period of the Mimas longitude libration due to a 4:2 mean motion resonance (MMR) with Tethys (Hedman et al. 2010); therefore, Tethys perturbs the orbit of Aegaeon through its interaction with Mimas.

Furthermore, variations in Aegaeon's eccentricity and inclination, not related to the CER, indicate a possible influence of other resonances, particularly the 7:6 inner Lindblad resonance (ILR), which resonant argument is given by $\varphi_{\mathrm{ILR}}=7 \lambda_{\text {Mimas }}-6 \lambda_{\text {Aegaeon }}-$ $\bar{\omega}_{\text {Aegaeon. }}$. This resonant argument librates, although not so tightly as the argument of the CER, with an amplitude of $\sim 90^{\circ}$ around zero (Hedman et al. 2010); therefore, the ILR could be inducing second or higher order perturbations, not well characterized so far.

Methone is a small moon of $\sim 1.45 \mathrm{~km}$ in diameter (Thomas et al. 2013). First reported by Porco et al. (2005), it is located at $\sim 194200 \mathrm{~km}$ from Saturn centre. It inhabits inside an arc of dust for which most probably it is the source (Sun et al. 2017). The arc's longitudinal extend is $\sim 10^{\circ}$, roughly centred at the small moon. Both Methone and its arc orbit near the 15:14 CER and the 15:14 outer Lindblad resonance (OLR) with Mimas (both resonances are separated by just 4 km; El Moutamid, Sicardy \& Renner 2014). Both resonant arguments of the 15:14 CER and OLR librate with amplitude $\sim 90^{\circ}$ around zero. However, the arc confinement is consistent with the maximum extension permitted by the 15:14 CER with Mimas alone (Hedman et al. 2009).

Anthe is a tiny moon of an estimated $\sim 0.5 \mathrm{~km}$ in diameter; a better define measurement has not been carried out to date, due to the lack of resolution in Cassini images (Thomas et al. 2013). Anthe is located at $\sim 197600 \mathrm{~km}$ from Saturn centre. It was first reported by Cooper et al. (2008), whom characterized its short-term dynamics. They found that Anthe orbits close to an 11:10 outer resonance with Mimas, with two librating arguments, one of which is associated with the 11:10 CER with Mimas, $\varphi_{\mathrm{CER}}=11 \lambda_{\text {Anthe }}-10 \lambda_{\text {Mimas }}-$ $\bar{\omega}_{\text {Mimas }}$, while the other one is given by $\varphi_{2}=11 \lambda_{\text {Anthe }}-10 \lambda_{\text {Mimas }}-$ $\bar{\omega}_{\text {Anthe }}-\Omega_{\text {Anthe }}+\Omega_{\text {Mimas }}$. Both periods of libration for these arguments are $761 \mathrm{~d}$ (Cooper et al. 2008) and librate with amplitude $\sim 90^{\circ}$ around zero. Likewise Aegaeon and Methone, Anthe lies at the centre of an arc of dusty material that extends for $\sim 20^{\circ}$, consistent with the confinement produced by the 11:10 CER with Mimas (Hedman et al. 2009).

Both arcs of Methone and Anthe have been deeply studied recently by Sun et al. (2017). They explored the evolution of $\mu \mathrm{m}-$ sized ejecta produced by collisions of interplanetary dust particles (IDPs) on the surfaces of the small moons. They then explored the dynamical evolution of the ejected particles considering an oblate Saturn, the gravitational perturbations of Mimas, Enceladus and Methone or Anthe, depending on the system, plus a number of non-gravitational effects, such as solar radiation pressure, Lorentz force and plasma drag. The authors are able to reproduce some of the properties of both arcs, such as their longitudinal extend and optical depth, although dust density in the simulations is an order of magnitude smaller compared to estimations derived from observations. According to Sun et al. (2017), particles smaller than 5 $\mu \mathrm{m}$ are quickly removed from the arcs due mainly to the action of plasma drag, while larger particles could survive up to $100 \mathrm{yr}$ in the arcs before recolliding with the parent small moon or leaving the arc.

Regarding Pallene, also first reported by Porco et al. (2005), it is a small moon of diameter $\sim 2.23 \mathrm{~km}$ (Thomas et al. 2013), orbiting at $\sim 212300 \mathrm{~km}$ from Saturn centre. It shares its orbit with a faint full ring of dusty material. This ring is only visible at extremely high phase angles, implying that it is formed by $\mu \mathrm{m}$-sized particles. The Pallene ring is very thin, with a vertical width of just $\sim 50 \mathrm{~km}$. For comparison, Pallene achieves vertical excursions of $1300 \mathrm{~km}$ above Saturn's equatorial plane due to its relatively large inclination. The ring is therefore tightly constrained to the orbital plane of Pallene, sharing the same inclination of the small moon (Hedman et al. 2009). Regarding their dynamics, Spitale et al. (2006) suggested that both the moon and its ring were probably in a 19:16 inner mean longitude resonance with Enceladus, with resonant argument given by $\theta=19 \lambda_{\text {Enc }}-16 \lambda_{\text {Pall }}-\bar{\omega}_{\text {Pall }}-2 \Omega_{\text {Pall }}$. Meanwhile, Callegari \& Yokoyama (2010) argued for a secular resonance relating Pallene and Mimas, with resonant argument given by $\theta=\bar{\omega}_{\mathrm{Pal}}-\bar{\omega}_{\mathrm{Mim}}-\Omega_{\mathrm{Mim}}+\Omega_{\mathrm{Pal}}$. Nonetheless, both arguments circulate, with different periods, suggesting that Pallene is most probably evolving out of any strong resonance. We provide some details in Sections 3 and 5 .

The short-term dynamics of the four small moons have been explored in a number of works, mainly those concerning their discovery and orbit determination from Cassini raw images. As we have recalled, Aegaeon, Methone and Anthe orbit near CER with Mimas, while Methone and Anthe also lie close to Lindblad resonances with Mimas. CER and Lindblad resonances are separated by just some $\mathrm{km}$ and are very close to the mean semimajor axis of Methone and Anthe. Motivated by this, El Moutamid et al. (2014) proposed a simplified analytical model, named CoraLin model, to explore the dynamics of test particles close to both CER and Lindblad eccentricity resonances (LER), which can be applied, under some assumptions, to these small moons. The CoraLin model is a simplification of the Hamiltonian for a restricted three body problem, where the large perturbing moon is eccentric and the test particle orbits near an MMR of the form $m+1: m$, where $m$ is an integer. The model depends upon just two free parameters. The first one, $D_{C}$, is related to the separation distance between the positions of the CER and LER, while the second parameter, $\epsilon_{L}$, is related to the mass and eccentricity of the perturbing satellite. $D_{C}=0$ means that both CER and LER lie at the same position, and the Hamiltonian is integrable in this case. For $D_{C}>2$, both resonances are separated enough as to not interfere with each other and the Hamiltonian is quasi-integrable. Intermediate cases, $0<D_{C}<2$ leads to strong coupling between the resonances, which in turn leads to a chaotic evolution of the orbits.

The results from applying the CoraLin model to the small moons, by inspecting the corresponding Poincare surfaces of section, suggest that Anthe is closer to the chaotic region than any of the other small moons. Methone is farther from the chaotic region than Anthe, while Aegaeon is the more stable, for being far enough from the chaotic region. Those small moons form a distinctively family of objects which dynamics is dominated by CER and LER coupling resonances (Cooper et al. 2015).

The only long-term exploration so far regarding the dynamics of the small moons (except for Aegaeon) was carried out by Callegari \& Yokoyama (2010). They performed $\sim 60000 \mathrm{yr}$ numerical integrations of the orbits of Methone, Anthe and Pallene under the influence of Saturn's oblateness until $J_{4}$, and the gravitational perturbations from Mimas, Enceladus, Tethys, Dione, Rhea 
and Titan. They found a high sensitivity on initial conditions for both Methone and Anthe, suggesting that their evolution could be chaotic, while Pallene seems to be very stable. The authors suggest a possible resonance involving the longitudes of pericentre and ascending nodes of Pallene and Mimas, however, as previously noted, such resonant argument does not librate, but circulates with a long period, as we will verify in Sections 3 and 5.

Regarding their physical characteristics, the Cassini images have revealed smooth surfaces for two moons, Methone and Pallene, for which there are enough resolution in the images. It is expected that both Aegaeon and Anthe share the same feature, i.e. a smooth surface; this is because of their proximity, size and likely same formation process. Moreover, since the discovery of the arc/ring structures associated with the small moons, the source of the micrometric material forming such structures has been assumed to be the escaped debris particles produced when IDP collided on the surface of the small moons. Due to their small size, their escape velocity is very low, letting almost all impact ejecta freely evolve out of the surface of the small moon into the arcs/ring. However, a considerable proportion of those dusty particles end up colliding again with the moons, smoothly depositing themselves on the surface of the small moons, thus being likely responsibly for the even surfaces observed. Numerical evidence for such scenario can be found in Sun et al. (2017).

In this paper, we explore the long-term evolution of the four small moons, exceeding any previous integration of their orbits. We also explore, with thousands of test particles, a wide region of the geometric phase space of semimajor axis, $a$, versus eccentricity, $e$, surrounding the small moons, to gain a global understanding of the dynamical behaviour and evolution of such regions where arcs/ring structures coexist.

This paper is organized as follows. In Section 2, we describe the frequency analysis technique and the simulations used in this work. Section 3 is devoted to the short-term evolution of the small moons, while in Section 4 we present several diffusion maps, based on frequency analysis, which characterize the dynamical behaviour of all the regions of interest. In Section 5, we present our main results regarding the long-term evolution of the small moons. Finally, in Section 6 we enumerate our main conclusions.

\section{METHODS AND NUMERICAL SIMULATIONS}

\subsection{Frequency analysis}

Frequency analysis is a powerful technique to quantify the weak chaotic behaviour of a dynamical system with arbitrary degrees of freedom. Since its original formulation by Laskar (1990, 1993), who develop it in order to prove the chaotic nature of the Solar system's secular evolution, it has been successfully applied to a wide variety of dynamical problems, going from planetary sciences (Nesvorný \& Morbidelli 1998; Robutel \& Laskar 2001) to galactic dynamics (Papaphilippou \& Laskar 1998; Valluri \& Merritt 1998), and even through fundamental particle physics (Nadolski \& Laskar 2003; Papaphilippou 2014).

The frequency analysis algorithm developed by Laskar looks for the fundamental frequencies of the system resulting from a numerical integration. A brief sketch of the method and, at the same time, a justification for its use can be stated as follows (Laskar, Froeschlé \& Celletti 1992): consider the Hamiltonian of an integrable dynamical system, $H(J, \theta)$; once the system is reduced to action angle variables, $\left(J_{j}, \theta_{j}\right)$, the Hamiltonian will depend only on the actions, $H(J, \theta)=H_{0}\left(J_{j}\right)$, where $j=1,2, \ldots, n$ for a system of $n$ degrees of freedom. We know that actions are constants of the motion, $\dot{J}_{j}=0$, while angles evolve according to

$\dot{\theta_{j}}=\frac{\partial H_{0}\left(J_{j}\right)}{\partial J_{j}}=v_{j}(J)$,

where the $v_{j}(J)$ are the fundamental frequencies of the motion.

In numerical experiments, we will rarely work on action angle variables, nonetheless, we can still consider some close related dynamical variables, say $z_{j}^{\prime}$, of the form: $z_{j}^{\prime}=J_{j}^{\prime} \exp \left(i \theta_{j}^{\prime}\right)=$ $f\left(z_{1}, z_{2}, \ldots, z_{n}\right)$, where $z_{j}=J_{j} \exp \left(i \theta_{j}\right)$; that is, any variable will be a certain function, $f$, close to unity, that depends on the actual actions and angles of the system. Although $\left(J_{j}^{\prime}, \theta_{j}^{\prime}\right)$ are not the action angle variables, the analysis of $z_{j}^{\prime}(t)$ will still give us the fundamental frequencies, $v_{j}$, since for periodic and quasi-periodic motions, these frequencies will remain constant.

If we recover the fundamental frequencies in different intervals of a numerical integration, we will be able to determine the existence of periodic motion, if frequencies remain almost constant, or on the contrary, if largely variable frequencies are found, the implication is an irregular, maybe chaotic motion. This is because, by inverting equation (1), we can state that $J=F\left(v_{1}, v_{2}, \ldots, v_{n}\right)$; therefore, variable frequencies imply variable actions.

We are not interested in recovering the analytical representation of particles orbits in our region of interest. On the contrary, we are interested in the variations of their motions due to different perturbations; thus, we focus on the global variations of the frequencies and not in their specific values. In this work, we calculate the main frequency, $v$, of the quantity $z_{j}^{\prime}=a(t) \exp (i \lambda(t))$ from a time series data set resulting from a numerical integration. To obtain the frequencies, we use the frequency-modified Fourier transform (FMFT) algorithm, described in Šidlichovský \& Nesvorný (1996), made publicly available by the author. ${ }^{1}$

According to Robutel \& Laskar (2001), the main frequency of $z^{\prime}(t)$ will be related to the mean motion, $n$, of the orbit. A full representation of $z^{\prime}(t)$ through frequency analysis would be

$z^{\prime}(t)=\alpha_{0} \exp \left(i v_{0} t\right)+\sum_{j}^{N} \alpha_{j} \exp \left(i v_{j} t\right)$,

where $v_{0}=n$ in the case of pure Keplerian motion. As this is not the case, both $\left|\alpha_{0}\right|$ and $v_{0}$ are close, but not equal, to the initial semimajor axis and mean motion, respectively. Nonetheless, the amplitudes of the following terms are 100-1000 times smaller for quasi-periodic trajectories; therefore, variations in the main frequency, $v_{0}$, will be enough to provide an estimation of the orbit's stability.

\subsection{Numerical simulations}

In order to obtain a global dynamical perspective of the region inhabited by Aegaeon, Methone, Anthe and Pallene, we performed short-term $(\sim 18 \mathrm{yr})$ numerical simulations of thousands of test particles that initially cover the entire $a$ versus $e$ geometric phase space from the orbit of Janus-Epimetheus to beyond the orbit of Enceladus. The choice of our integration time relies on a compromise, between the large volume of data produced and the requirement of enough orbital periods to accurately calculate the main frequencies for our analysis. In $\sim 18 \mathrm{yr}$, Mimas performs $\sim 6950$ orbital revolutions around Saturn, while Enceladus, close to the exterior limit

\footnotetext{
${ }^{1}$ https://www.boulder.swri.edu/ davidn/fmft/fmft.html
} 
Table 1. Summary of physical parameters of the moons.

\begin{tabular}{lcccc}
\hline Name & $\mathrm{GM}\left(\mathrm{km}^{3} \mathrm{~s}^{-2}\right)$ & $\rho\left(\mathrm{g} \mathrm{cm}^{-3}\right)$ & $R_{m}(\mathrm{~km})$ & Reference \\
\hline Janus & $1.2656324971531704 \mathrm{E}-01$ & 0.63 & 89.2 & Thomas et al. (2013) \\
Epimetheus & $3.512421991952764 \mathrm{E}-02$ & 0.64 & 58.2 & Thomas et al. (2013) \\
Aegaeon & $1.2606299971854121 \mathrm{E}-09$ & 0.54 & 0.33 & Thomas et al. (2013) \\
Mimas & $2.502784093954375 \mathrm{E}+00$ & 1.15 & 198.2 & Cooper et al. (2015) \\
Methone & $6.261297469640338 \mathrm{E}-08$ & 0.31 & 1.45 & Thomas et al. (2013) \\
Anthe & $2.334499994563023 \mathrm{E}-08$ & 0.35 & 0.5 & Thomas et al. (2013) \\
Pallene & $1.7980185560068351 \mathrm{E}-07$ & 0.25 & 2.23 & Thomas et al. (2013) \\
Enceladus & $7.211597878640501 \mathrm{E}+00$ & 1.6 & 252.6 & Cooper et al. (2015) \\
Tethys & $4.121706150116760 \mathrm{E}+01$ & 0.956 & 537.5 & Cooper et al. (2015) \\
\hline
\end{tabular}

Table 2. Saturn parameters.

\begin{tabular}{lcc}
\hline Constant & Value & Reference \\
\hline $\mathrm{GM}\left(\mathrm{km}^{3} \mathrm{~s}^{-2}\right)$ & $3.793120706585872 \mathrm{E}+07$ & Cooper et al. (2015) \\
$R_{S}(\mathrm{~km})$ & 60,330 & Cooper et al. (2015) \\
$J_{2}$ & $1629.054382 \mathrm{E}-05$ & Hedman et al. (2010) \\
$J_{4}$ & $-93.6700366 \mathrm{E}-05$ & Hedman et al. (2010) \\
$J_{6}$ & $8.6623065 \mathrm{E}-05$ & Hedman et al. $(2010)$ \\
\hline
\end{tabular}

of our maps, performs 4780 revolutions; that is, we obtain enough orbital revolutions for all particles for an accurate recovery of their frequencies.

Test particles are subject to gravitational perturbations from the oblateness of Saturn up to $J_{6}$ in zonal harmonics, plus the five largest moons of the region, namely Janus, Epimetheus, Mimas, Enceladus and Tethys, in a first set. In later simulations, we consider the gravitational perturbations from Saturn and the large five moons, as well as the gravitational perturbations from the four small moons. After the global exploration, we zoomed in towards those regions closer to each small moon, performing simulations that include thousands of test particles covering a smaller patch of the geometric $a$ versus $e$ phase space, but in finer steps. We provide the details for each simulation along with the results in the next section.

In Section 5 , the results for a single long-term $\left(1 \times 10^{5} \mathrm{yr}\right) \mathrm{nu}-$ merical integration are reported, where only the interaction between the five large moons and the four small moons, orbiting an oblate Saturn, is considered.

All of the numerical simulations presented in this work were performed using the Bülirsch-Stöer integrator from the MERCURY package (Chambers 1999), where a toleration accuracy parameter was set to $10^{-12}$ and an initial time step of $0.1 \mathrm{~d}$ was used. Most of the simulations were performed at the Saturn Cluster belonging to the Group of Planetology and Orbital Dynamics of the Mathematics Department of the São Paulo State University (UNESP).

The physical parameters of all the nine different moons used throughout this work are shown in Table 1, where GM is the gravitational parameter, $\rho$ is the bulk density of the body and $R_{m}$ the mean radius. In Table 2, we show the parameters of Saturn.

\section{SHORT-TERM DYNAMICS}

To test the robustness of the initial conditions used in all of our simulations, we performed a detailed short-term integration, lasting for $24 \mathrm{yr}$, in order to reproduce the resonant dynamics of Aegaeon, Methone and Anthe, as has been found by previous authors. This simulation includes the nine moons presented in Table 1, plus Saturn and its gravity coefficients given in Table 2. The initial conditions for all the bodies were taken from the Horizons website for the Julian Day 2457 601.5, which corresponds to the date 2016 August 1. The geometric elements used as initial conditions for the nine objects that constitute the $n$-body part of the simulations are shown in Tables 3 (for the five large moons) and 4 (for the four small moons), where all variables have their usual meaning of geometric semimajor axis, $a$, geometric eccentricity, $e$, geometric inclination, $i$, longitude of pericentre, $\bar{\omega}$, longitude of the ascending node, $\Omega$, and mean longitude, $\lambda$. In Tables 3 and $4, D_{\text {Mim }}$ stands for the normalized geometric semimajor axis of Mimas, $\mathrm{D}_{\text {Mim }}=1$, used as a reference distance unit throughout this work. In physical units, $\mathrm{D}_{\text {Mim }}=1.85539 \times 10^{5} \mathrm{~km}$.

The most important constraint for the dynamics of the small moons is the resonant dynamics associated with several resonances, amongst them are the CER and LER with Mimas. In Fig. 1, we show the short-term evolution of the resonant arguments for Aegaeon, Methone and Anthe resonances. In addition, we plotted the evolution of the two arguments associated with the quasi-resonances of Pallene with Enceladus and Mimas, as suggested by Spitale et al. (2006) and Callegari \& Yokoyama (2010), respectively.

Hedman et al. (2010) found that Aegaeon is trapped in the 7:6 CER with Mimas, with a libration argument $\phi_{\text {Aeg }}=7 \lambda_{\mathrm{Mim}}-$ $6 \lambda_{\text {Aeg }}-\bar{\omega}_{\text {Mim }}$, a period of libration around $180^{\circ}$ of $\sim 1264 \mathrm{~d}$ and amplitude of $\sim 10^{\circ}$. We found (the top panel of Fig. 1) a libration period of $\sim 1252.5 \mathrm{~d}$, a difference of only 0.9 per cent with the previous result and the same libration amplitude of $10^{\circ}$ around $180^{\circ}$.

For Methone, both Spitale et al. (2006) and Jacobson et al. (2006) found a libration argument $\phi_{\text {Met }}^{\mathrm{LER}}=15 \lambda_{\mathrm{Met}}-14 \lambda_{\mathrm{Mim}}-\bar{\omega}_{\mathrm{Met}}$, a period of libration of $\sim 450 \mathrm{~d}$ and an amplitude of the residual longitude of $\sim 5^{\circ}$. This argument corresponds to the $15: 14$ LER with Mimas. Nonetheless, Hedman et al. (2009) also found that the argument associated with the 15:14 CER with Mimas, $\phi_{\text {Met }}^{\mathrm{CER}}=15 \lambda_{\mathrm{Met}}-14 \lambda_{\mathrm{Mim}}-\bar{\omega}_{\text {Mim }}$, also librates with the same period and amplitude as the one associated with the LER. This means than Methone is perturbed by both the CER and the LER with Mimas, which are separated by just $4 \mathrm{~km}$. We found that both arguments for the CER (blue curve in the second panel of Fig. 1) and the LER (red curve in same panel) of Methone effectively librate, and we have normalized the two arguments for them to librate around zero, in order to compare their amplitudes, which is around $90^{\circ}$. Their period of libration is of $\sim 515.7 \mathrm{~d}$, a difference of $\sim 14$ per cent with previous results. Such differences could be the result of the updated orbital data and masses we have used. An evidence in this sense is the recent result found by Sun et al. (2017) for the Methone libration period of the CER. They found this period to be $\sim 520 \mathrm{~d}$ and an amplitude of the residual longitude of $\sim 5^{\circ}$. The result of Sun et al. (2017) is in better agreement with ours, since they used updated 
Table 3. Initial conditions in geometric elements for the large moons.

\begin{tabular}{lccccc}
\hline Parameter & Janus & Epimetheus & Mimas & Enceladus & Tethys \\
\hline$a\left(\mathrm{D}_{\text {Mim }}\right)$ & 0.816224821915373 & 0.816481357153813 & 1.00001997053844 & 1.282926463121003 & 1.588211135154469 \\
$a(\mathrm{~km})$ & 151441.5372 & 151489.1345 & 185542.7053 & 238032.8930 & 294675.1058 \\
$e$ & 0.006783798913451 & 0.009648290023497 & 0.01935393988488 & 0.004717750162711 & 0.000318152308649 \\
$i\left({ }^{\circ}\right)$ & 0.163561569028246 & 0.351528831169069 & 1.56783494576081 & 0.004011975196723 & 1.091284647139937 \\
$\bar{\omega}\left({ }^{\circ}\right)$ & 6.132967855641644 & 116.348018800562642 & 88.06783652593947 & 57.40490801677754 & 29.38263145675355 \\
$\Omega\left({ }^{\circ}\right)$ & 6.369960248480534 & 44.525088430924164 & 239.785329147143301 & 337.68709939428453 & 141.711912205570002 \\
$\lambda\left(^{\circ}\right)$ & 129.813146812610796 & 263.577236051360842 & 318.616779106079946 & 217.90585212208282 & 271.834917459084238 \\
\hline
\end{tabular}

Table 4. Initial conditions in geometric elements for the small moons.

\begin{tabular}{lcccc}
\hline Parameter & Aegaeon & Methone & Anthe & Pallene \\
\hline$a\left(\mathrm{D}_{\text {Mim }}\right)$ & 0.90274553988027 & 1.046749724679189 & 1.065312819759956 & 1.144147730931239 \\
$a(\mathrm{~km})$ & 167494.5047 & 194212.8971 & 197657.0752 & 212284.0258 \\
$e$ & 0.000312629006216 & 0.000781255131993 & 0.000980088315003 & 0.003986601194199 \\
$i\left({ }^{\circ}\right)$ & 0.002368099675087 & 0.010191700663056 & 0.018728031233752 & 0.181964040454351 \\
$\bar{\omega}\left({ }^{\circ}\right)$ & 240.784600532626655 & 101.055122930558355 & 196.379681976898155 & 320.254677876382459 \\
$\Omega\left({ }^{\circ}\right)$ & 246.49085511672857 & 150.999808094857514 & 115.172628200709838 & 131.420435093503784 \\
$\lambda\left({ }^{\circ}\right)$ & 325.21506542212154 & 205.403404370634803 & 159.569919065624163 & 293.49347042844056
\end{tabular}

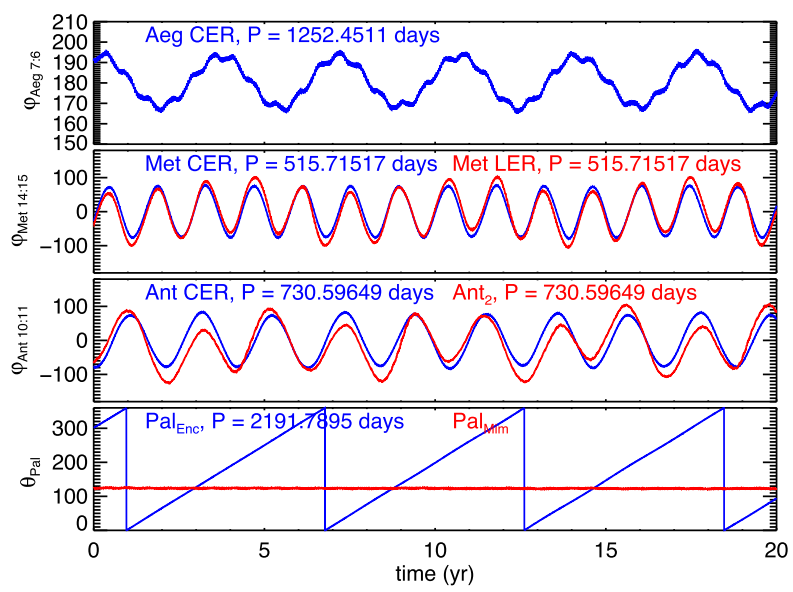

Figure 1. Short-term evolution of different resonant arguments of the small moons. Each panel shows the evolution for $20 \mathrm{yr}$ of the arguments associated with the resonances in which the small moons are known or suggested to be trapped. The top panel for Aegaeon, second for Methone, third for Anthe and the bottom panel for Pallene. See the text for details.

data from the Horizons system of the Jet Propulsion Laboratory, as we did.

In the case of Anthe, Cooper et al. (2008) found two resonant arguments that librate, one is the associated with the 11:10 CER with Mimas, $\phi_{\text {Ant }}=11 \lambda_{\text {Ant }}-10 \lambda_{\text {Mim }}-\bar{\omega}_{\text {Mim }}$, and the other argument is given by $\phi_{\mathrm{Ant}_{2}}=11 \lambda_{\mathrm{Ant}}-10 \lambda_{\mathrm{Mim}}-\bar{\omega}_{\mathrm{Ant}}-\Omega_{\mathrm{Ant}}+\Omega_{\mathrm{Mim}}$. For both arguments, Cooper et al. (2008) found a period of libration of $\sim 761 \mathrm{~d}$ and an amplitude of $78^{\circ}$. We find for both arguments (blue and red curves in the third panel of Fig. 1) a period of $\sim 731$ $\mathrm{d}$, a difference of just 4 per cent with the previous result, with an amplitude of libration of $\sim 90^{\circ}$. Hedman et al. (2009) and Sun et al. (2017) argue for the 11:10 CER of Anthe with Mimas to be responsible for the confinement of the Anthe's arc, being then the most relevant for the evolution of the moon and its environment.

Finally, although Pallene is not in resonance neither with Mimas or Enceladus, both Spitale et al. (2006) and Callegari \& Yokoyama (2010) suggest two different arguments that circulate and could indicate a quasi-resonance of Pallene with any of those large moons. First, Spitale et al. (2006) suggest the argument: $\theta_{\text {Pall }}^{\text {Enc }}=19 \lambda_{\text {Enc }}-16 \lambda_{\text {Pall }}-\bar{\omega}_{\text {Pall }}-2 \Omega_{\text {Pall }}$, a $19: 16$ inner mean longitude resonance between Pallene and Enceladus, as responsible for the long-term variations in the orbital elements of Pallene. On the other hand, Callegari \& Yokoyama (2010) argue for an argument given by $\theta_{\text {Pall }}^{\mathrm{Mim}}=\bar{\omega}_{\text {Pal }}-\bar{\omega}_{\text {Mim }}-\Omega_{\text {Mim }}+\Omega_{\text {Pal }}$, which implies a relation between Pallene and Mimas, based on the high stability found by the authors in their long-term simulations. They obtain a period of circulation of $\sim 4400 \mathrm{yr}$. We find that in fact both cited arguments circulate, where the one involving Pallene and Enceladus has a period of circulation of $\sim 2192 \mathrm{~d}$ (blue curve in bottom panel of Fig. 1), while the other argument, involving Mimas and Pallene, although at first glance could seem as librating (see the red curve in Pallene's panel), it actually circulates but with a much larger period, not visible in this short-time-scale plot. We show the circulation of the second argument in Section 5, for which we find a period of $\sim 4762.21 \mathrm{yr}$, a difference of $\sim 7.6$ per cent compared with Callegari \& Yokoyama (2010) work.

\section{A GLOBAL DYNAMICAL PERSPECTIVE THROUGH FREQUENCY ANALYSIS MAPS}

\subsection{The full region around Mimas}

The aim of this section is to delineate the lookalike of a wide dynamical region around Mimas, going from 0.7 to $1.3 \mathrm{D}_{\mathrm{Mim}}$, to better understand the influences that can drive the evolution of the small moons and their ring/arcs. In Fig. 2, we plot the location of all first- and second-order MMRs with Janus, Mimas, Enceladus and Tethys present in this region of the Saturnian system. All this resonances are somewhat responsible for the shaping of structures, cleaning or maintaining of the particles in the region, which are subject to gravitational forces only. Although it is well known that all Aegaeon, Methone, Anthe and their associated arcs lie in CER with Mimas, here we provided a global framework for further detailed explorations near any specific MMR of interest, where other kinds of dynamics could better fit to the particular case, such as corotation or Lindblad resonances. 


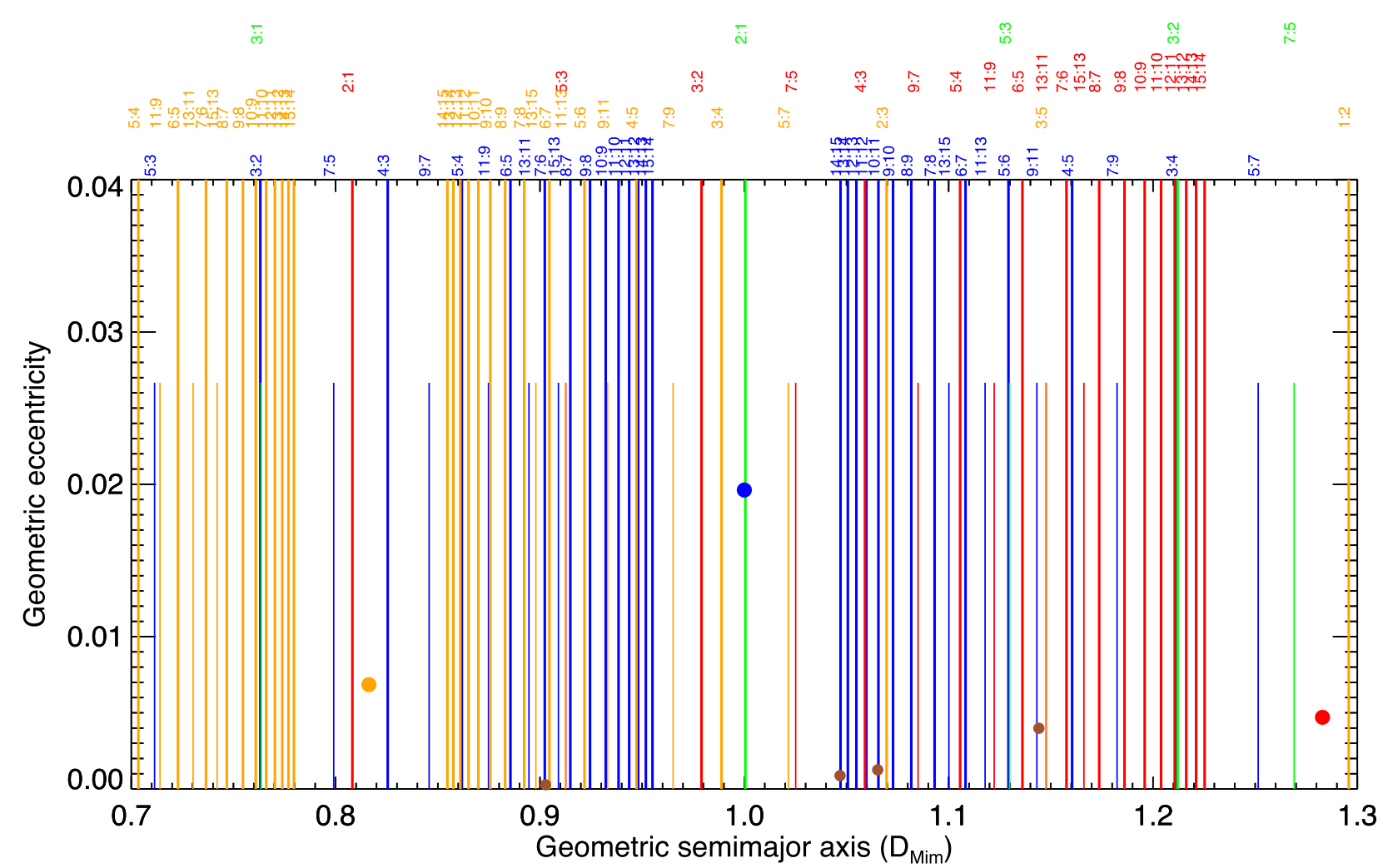

Figure 2. Location of first and second order MMRs in the region. We plot coloured vertical lines at the position of interior and exterior first order MMRs (thick lines from bottom to top) and second order MMRs (thin lines from bottom to middle), up to the ratio $p / q=15 / 14$, inside the region of phase-space, of geometric semimajor axis versus geometric eccentricity, explored in this work. Colour code is as follows: orange lines stand for MMRs with Janus (moon location also shown as filled orange circle), blue lines for MMRs with Mimas (moon as blue circle), red lines for MMRs with Enceladus (moon as red circle), and green lines for MMRs with Tethys (not shown in the plot). The corresponding MMR ratios are shown in an adequate colour at the top of the figure. Small filled brown circles indicate the location of the four small moons, from left to right: Aegaeon, Methone, Anthe, and Pallene.

The global dynamical picture of the full region was obtained from the detailed numerical integration during $\sim 18$ yr of 11000 test particles distributed in a rectangular grid, covering the geometric phase space of $a$ versus $e$. In $a$, initial conditions are taken from 0.7 to 1.3 $\mathrm{D}_{\mathrm{Mim}}$ in steps of $6.0 \times 10^{-4}$. In $e$ we cover from 0 to 0.04 in steps of $4 \times 10^{-3}$. In all this work, we explore the evolution of particles initially in the Saturn's equatorial plane (thus all test particles have initially zero inclination). As for the remaining orbital elements of each particle, $\bar{\omega}, \Omega$ and $\lambda$, they are set to zero, following the approach of Robutel \& Laskar (2001) in their global exploration of the dynamics of the Solar system. Indeed, the variations of the angular elements $(\bar{\omega}, \Omega$, and $\lambda)$ do not change significantly the results for the diffusion of the particles outside resonances (which constitutes most of the dynamical space in all our experiments). Such variations lead only to different capture rates for particles which are initially close to MMRs and therefore are subject to get trapped. In this sense, angles' values are important mainly because they determine the width of the libration regions of MMRs. Nonetheless, in our case, as we only explored very low eccentricities, the width of MMRs is, as we will see, very thin, thus the contribution from variations of the angles is negligible.

All test particles are subject to the non-spherical potential of Saturn up to an order $J_{6}$ in zonal harmonics, plus the gravitational perturbations of the five large moons close to the region, namely Janus, Epimetheus, Mimas, Enceladus and Tethys, in the first set. For the second set, we also added the gravitational perturbations from Aegaeon, Methone, Anthe and Pallene. To account for the rapid orbital oscillations due to the oblateness of Saturn, the state vectors resulting from the integrations are everywhere transformed to geometric elements following Renner \& Sicardy (2006).

In order to construct the frequency analysis map, we explore the evolution of the quantity $z^{\prime}(t)=a(t) \exp (i \lambda)$, for each particle, where $a$ is the geometric semimajor axis and $\lambda$ is the mean longitude of the orbit. We have $T \sim 9 \mathrm{yr}$, equivalent to half the total integration time. We applied the FMFT (Šidlichovský \& Nesvorný 1996) over $z^{\prime}(t)$ on the adjacent time intervals, $[0, T]$ and $[T, 2 T]$, in order to compare the main frequencies of $z^{\prime}(t), v_{1}$ and $v_{2}$, from each time interval.

Since we are interested in the global dynamical structure of the region, we focus on the diffusion parameter, $D$, for each particle, defined following Robutel \& Laskar (2001) and Correia et al. (2005), as

$D=\frac{\left|v_{1}-v_{2}\right|}{T}$.

The $D$ parameter provides a measure of the particle's orbit stability. Some particles do not survive the whole simulation and we do not compute $D$ for them. For unstable particles, which nonetheless survive until the end of the simulation, the difference $\left|v_{1}-v_{2}\right|$ will be significant, leading to large values of $D$. On the contrary, for stable particles the same difference will be small, leading to very small values of $D$. An estimation of a diffusion time-scale, $t_{\text {Diff }}$, that is, an estimation of the time required until an appreciable change in the $n$ of the particle is observed, or the time required for a 


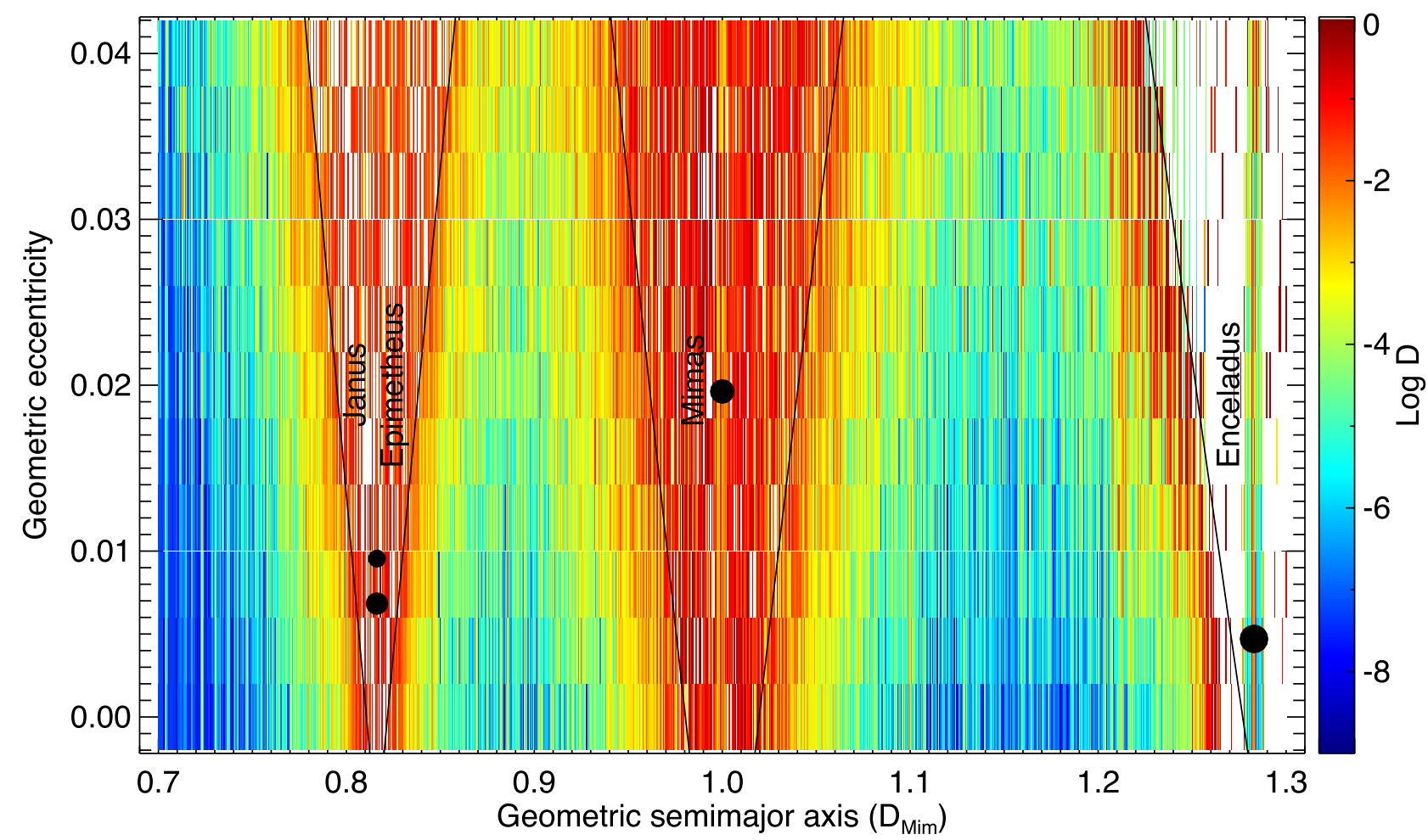

Figure 3. Diffusion map for the full region of interest. For this map, along with test particles, only the five large moons were included in the simulation, although Tethys is not shown due to its farther location. The diffusion parameter, $D$, is obtained from equation (3) after a frequency analysis is performed for each test particle. A rectangle, coloured according to the value of $\log (D)$, is plotted centred at each initial condition in the phase-space plane of geometric semimajor axis versus geometric eccentricity. Bluest regions correspond to the more stable orbits, while redder zones correspond to unstable trajectories. Some particles do not survive the whole simulation and for them we plotted a white rectangle, same colour as the background.

diffusion of the orbit in the radial direction, can be crudely estimated as $t_{\text {Diff }}=(D P)^{-1} \mathrm{yr}$, where $P$ is the period of the orbit in yr.

The FMFT algorithm let us to measure with confidence the variations of $v_{1}$ and $v_{2}$, in the very short-time integrations performed. In order to characterize the dynamical region of interest, we have plotted, in a colour scale, the $\log (D)$ for each particle, according to its initial position in the geometric $a$ versus $e$ phase-space plane. The resulting map of the entire region is shown in Fig. 3.

To highlight the relevance of the small moons over their environment, an analogous map to that of Fig. 3 is shown in Fig. 4. This map was obtained from the integration of the same set of test particles subject to the gravitational perturbations from the five large moons, plus the four small moons: Aegaeon, Methone, Anthe, Pallene and an oblate Saturn.

The maps presented in Figs 3 and 4 show the general dynamical characteristics of the region. In both figures, black lines delimit the position of constant pericentre and apocentre of test particles at the positions of the apocentre and pericentre of each major body, respectively. In Fig. 3, the positions of the four large moons are pointed by filled black circles. Near the coorbital moons Janus-Epimetheus, the constant pericentre and apocentre lines are those corresponding to the position of Janus' apocentre and pericentre, respectively, as it is the more massive moon of the two coorbitals. For Mimas, both curves of constant apocentre and pericentre are plotted. For Enceladus, only the line delimiting the constant apocentre of particles at the position of Enceladus' pericentre is shown. The redder colours in both maps are enclosed by such black lines. Particles that enter, or that are initially located inside such regions, are strongly perturbed by the major bodies, resulting in a major probability for them to collide with the corresponding moon, thus being lost from the simulation. In the map of Fig. 3, most particles inside the Enceladus region are removed before the end of the simulation, while much less particles are removed inside the regions of Mimas and Janus-Epimetheus. However, some of the particles inside the Enceladus region survived, remarkably those that are coorbital with the large moon. A small patch of stability is also seen for particles of small eccentricity, coorbital with Mimas, while no evident coorbital stability region is seen inside the Janus-Epimetheus region. Outside of the regions delimited by black lines, the more stable particles are those of small eccentricity expected, since they do not cross the orbit of any large moon.

Regarding particle collisions with the large moons we see that, for the simulation shown in Fig. 3, due to the Mimas larger eccentricity, compared to that of Enceladus or Janus and Epimetheus, it takes longer time for Mimas to clean up its region despite its large size. From the 1454 particles initially inside the Mimas region, only $\sim 12$ per cent of them collided with the large moon. The surviving particles have an average $\log (D)=-1.4091149$. This implies a diffusion time of $t_{\text {Diff }}=9.946 \times 10^{3} \mathrm{yr}$, considering the orbital period of Mimas. For Enceladus, we found 885 particles initially inside its region, out of which almost 70 per cent collided, and the surviving particles are mainly coorbitals with the large moon. The surviving particles have an average $\log (D)=-3.2606267$, which implies a $t_{\text {Diff }}=4.857 \times 10^{5} \mathrm{yr}$, considering the period of Enceladus. Finally, in the region dominated by Janus-Epimetheus, out of the 806 particles initially inside the corresponding region, 


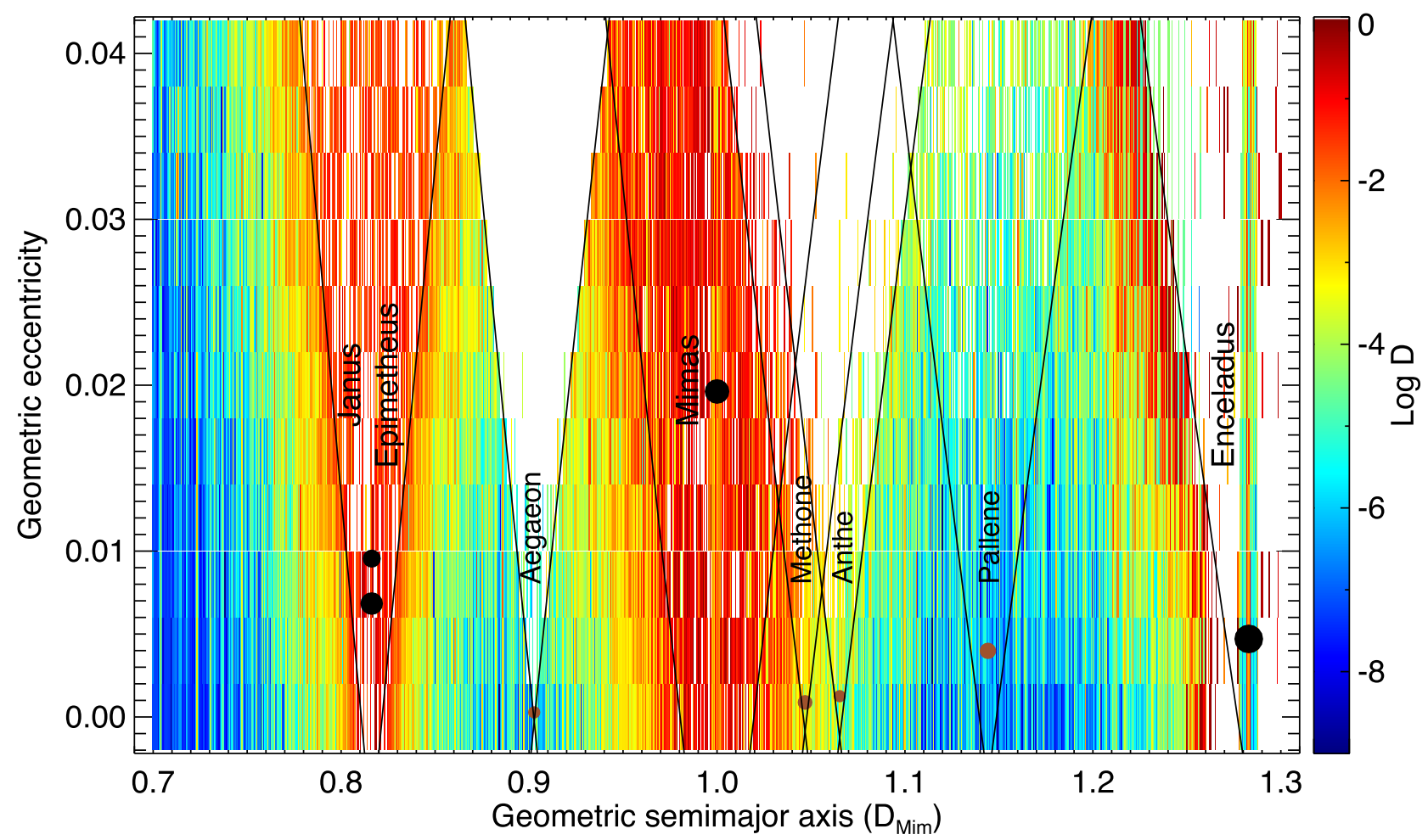

Figure 4. Diffusion map of the region of interest including all nine moons as $n$-bodies. As in Fig. 3, scale coloured rectangles are plotted for each initial condition according to the $D$ value of the particle. Wider white regions, where particles are removed before the end of the simulation, are now present due to collisions with the small moons.

around 36 per cent collided with any of the large moons. The average $\log (D)=-1.5613890$ of the surviving particles leads to a diffusion time of $t_{\text {Diff }}=1.915 \times 10^{4} \mathrm{yr}$, by considering the period of Janus.

In the simulation shown in Fig. 4, Aegaeon, Methone, Anthe and Pallene were included as $n$-bodies. The positions of the four small moons are indicated by brown circles in the map. All lines for constant pericentre and apocentre of test particles at the positions of apocentres and pericentres of the large and small moons are shown in black solid lines. In this case, a greater number of particles is removed from the collision regions (those enclosed by solid black lines) leading to the appearance of big white zones. As in the previous case, inside these collision regions, some coorbital particles survive, mainly with Enceladus and with Mimas to a lesser extent.

The results from the map of Fig. 4 suggest that the small eccentricity of Aegaeon, Methone and Anthe let them to better clean up their orbits in a short period of time $(\sim 18 \mathrm{yr})$, when compared to the much more massive Janus, Epimetheus or Mimas. Nonetheless, inclinations likely play also an important role for the clearing ability of the moons. To test this, we perform a simulation with the same conditions of that of Fig. 4, but this time with the initial inclinations of all the nine moons set to zero. The result is shown in the map of Fig. 5. Clearly, when the moon lies in the orbital plane, it better clear its path, this is manifest in the large white regions inside the black lines limiting the regions of Janus-Epimetheus and Mimas. Moreover, the coorbital stability region is clearly visible now for Mimas, and two high eccentricity coorbitals with Janus-Epimetheus also survive. In the case of Aegaeon, Methone and Anthe, the changes are insignificant as all they had from the beginning a small inclination $\left(<0.02^{\circ}\right)$. The same lack of change is observed for Enceladus, which has an $i \approx 0.004^{\circ}$.
Therefore, moons of small eccentricity and inclination clean their orbits efficiently, regardless their mass (see Aegaeon, Methone, Anthe and Enceladus), and coorbital particles with the lowly inclined moons are more likely to survive. On the other hand, moons with larger eccentricities and inclinations require longer times for the clearing of their orbits despite their large masses (see JanusEpimetheus, Mimas and Pallene), and coorbital particles are not likely to coexist with them due to a large inclination. Interestingly for Pallene from the previous exercises, we have gained an important hindsight about the high stability of the region surrounding this small moon. The combination of a small mass and a large inclination $\left(\sim 0.182^{\circ}\right)$ makes Pallene a weak perturber and inefficient clearer of its region.

In order to explore in detail the dynamical environment of the small moons, we mapped the geometric phase-space around them, zooming from the general view into three finer maps, one around Aegaeon, a second around Methone and Anthe, and one more around Pallene. Although the simulations last the same amount of time as the ones describe earlier, the coverage of $a$ and $e$ is far better. We describe the initial conditions used for each map in the next subsections.

\subsection{The dynamical environment of Aegaeon}

To get a better understanding of the dynamical evolution of Aegaeon and particles nearby, like the ones forming the $\mathrm{G}$ ring, we perform the numerical integration of the orbits of 11000 test particles, covering a small patch of the geometric $a$ versus $e$ phase space around the small moon. In $a$ we cover from 0.85 to $0.95 \mathrm{D}_{\text {Mim }}$ in steps of $1.0 \times 10^{-4}$. In $e$ we cover from 0.0 to 0.02 in steps of 


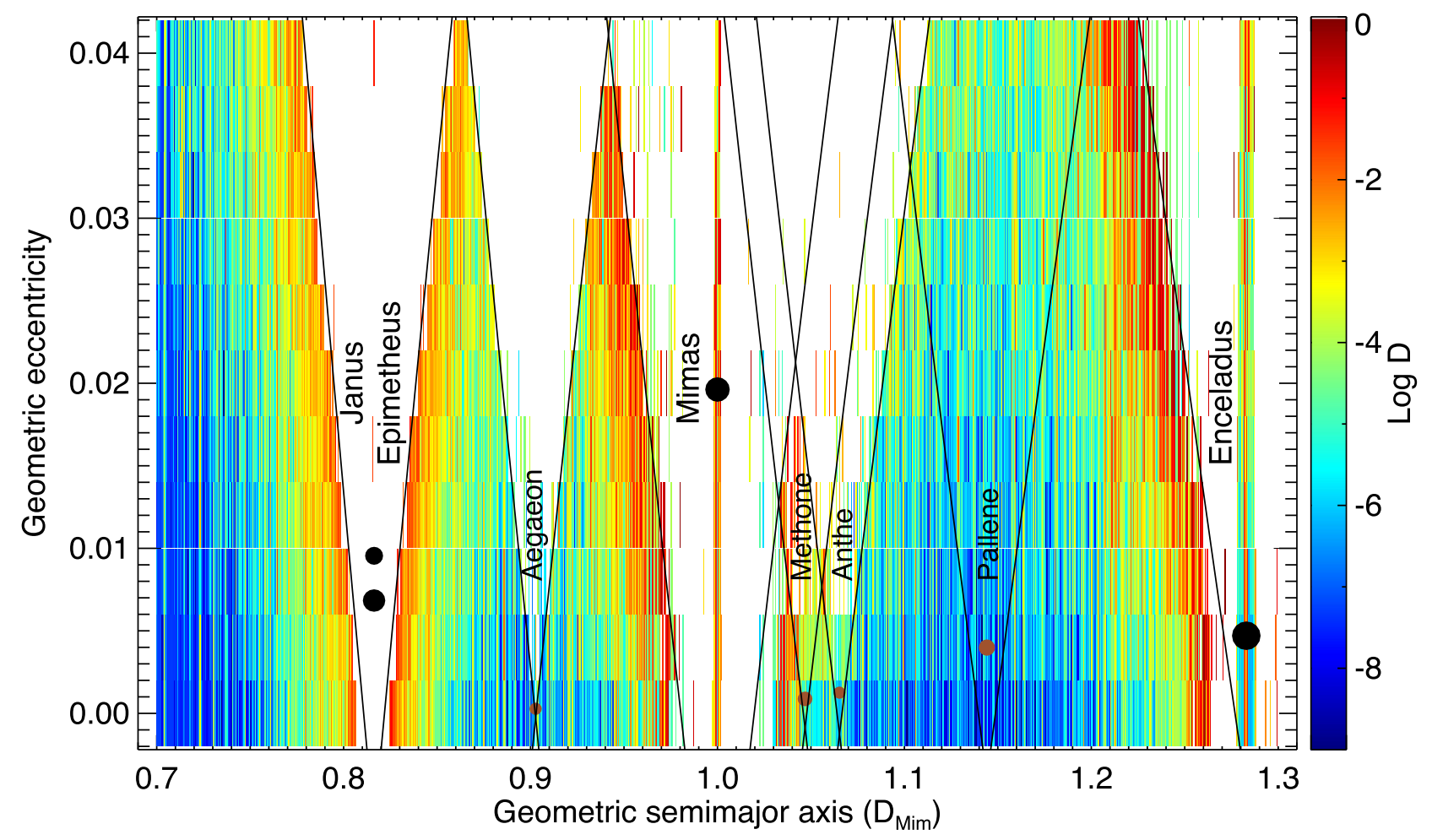

Figure 5. Same as Fig. 4 but with the initial inclination of all moons set to zero.

$2 \times 10^{-3}$. Initial inclination and the angles $\bar{\omega}, \Omega$ and $\lambda$ are all set to zero. Test particles are subject to gravitational perturbations from the oblateness of Saturn up to $J_{6}$, the five large moons of the global case described earlier, and Aegaeon, included as a massive body of $1.89 \times 10^{13} \mathrm{~g}$, assuming a bulk density of $0.54 \mathrm{~g} \mathrm{~cm}^{-3}$ (Thomas et al. 2013). The resulting diffusion map is shown in Fig. 6.

The average location of Aegaeon is shown in the map of Fig. 6 by a filled brown circle. This location is close but not equal to the location of the 7:6 MMR, as Aegaeon librates in the 7:6 CER with Mimas. The long-term dynamics of Aegaeon in this resonance will be further analysed in Section 5. In this map, black solid lines indicate the constant apocentre and pericentre distances of particles, at the position of Aegaeon's pericentre and apocentre, respectively. Most of the particles inside this region end up colliding with the small moon before the end of the simulation, which results in a wide white triangle feature. Initially, 2042 particles were inside the region enclosed by solid black lines, while just 303 of them survived, i.e. almost 85 per cent of the particles collided with Aegaeon in just $\sim 18$ yr. In average, surviving particles have $\log (D)=-4.6857625$, which implies a $t_{\text {Diff }}=2.192 \times 10^{7} \mathrm{yr}$.

As we can see from Fig. 2, a great number of first- and secondorder MMRs, mainly with Janus and Mimas, populate the region around Aegaeon. The evenly coloured vertical features in the map of Fig. 6, both interior and exterior to the orbit of Aegaeon, are related to any of these MMRs. To identify the strongest resonances, and therefore particles librating inside them, we make use of the frequency curve (Robutel \& Laskar 2001). In Fig. 7, we show the frequency curve, i.e. the ratio of the particle's mean motion over Mimas mean motion, $n / n_{\text {Mim }}$, versus the initial particle geometric $a$. MMRs are characterized for the flatness of the frequency curve due to a constant $n / n_{\text {Mim }}$ ratio of nearby particles of different $a$; this facilitates the identification of librating particles. In the curve of Fig. 7, we point out (with arrows in the figure) the presence of the next first-order MMRs: 6:5, 7:6, 8:7, 9:8, 10:9, 11:10, 11:12 and 13:12; although the flatness of the frequency curve associated with the mentioned resonances is barely visible, some particles cause the curve to flatten, making possible their identification. To exemplify the way how frequency curve flats in resonances, in Fig. 7 we zoomed into a region very close to the 7:6 MMR with Mimas, where a number of particles maintain a constant ratio $n / n_{\mathrm{Mim}}=7 / 6$. We note that the small maximum eccentricity covered by our grid of initial conditions accounts for the width of the resonant region to be very small, thus making difficult for such regions to stand out from the Keplerian trend of the rest of the frequency curve.

It is in this sense that the resonant regions are better recognized directly from the diffusion map of Fig. 6. In the map, we have plotted as black rectangles the resonant particles with Mimas identified from the frequency curve, i.e. the particles for which the ratio $n / n_{\text {Mim }}$ is equal to the corresponding MMR ratio $p / q$. Nonetheless, apart from the first-order MMRs with Mimas, other homogenous vertical regions are clearly recognizable, and other resonances can be related to them. For this, we have indicated at the top of the figure the corresponding ratios at the position of first- and second-order MMRs with Janus, in orange, and with Mimas, in blue. Such resonances could not be as strong as first-order MMRs with Mimas; however, their influence is imprint in the homogeneous $D$ parameter they produced over particles of different eccentricities.

We highlight the importance of MMRs on the evolution of test particles, even as there are not known structures or particles in such regions in the real system, i.e. outside the edges of the $\mathrm{G}$ ring. If ever a population of particles inhabited some of the stable regions currently empty, then it needs to be an explanation, other than the pure gravitational evolution, for the lost of such particles. Nongravitational forces are a likely explanation, given that particles 


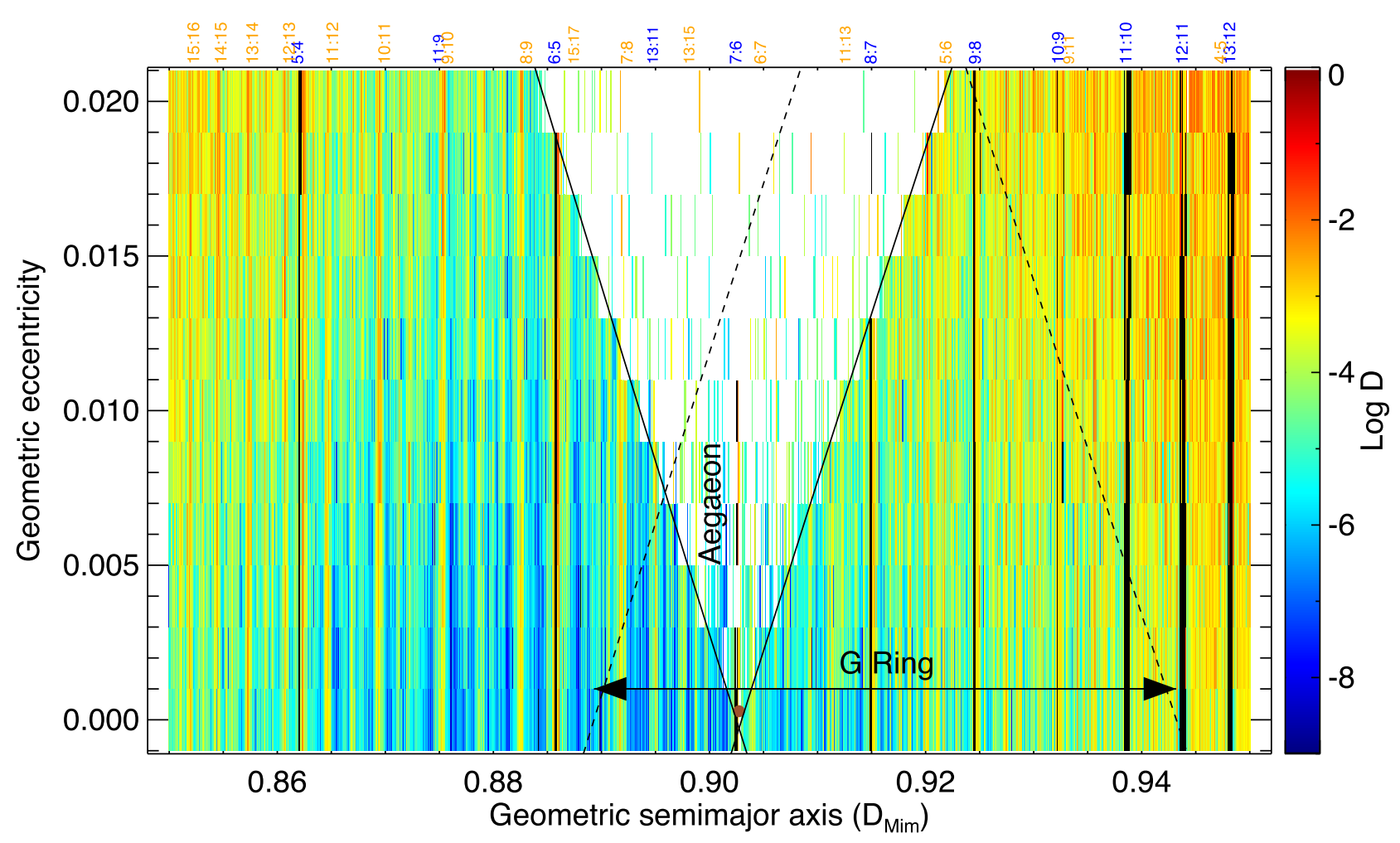

Figure 6. Diffusion map for the Aegaeon region. The location of Aegaeon is indicated by a brown circle. Solid black lines stand for the pericentric and apocentric distances of particles at the apocentre and pericentre distances of Aegaeon, respectively. Most particles inside this region collide with Aegaeon; however, some particles survived, mainly those with the smaller eccentricity. Resonant particles in first-order MMRs with Mimas are coloured in black. Labels for first- and second-order MMR ratios are shown at the top of the figure to indicate the location of the respective resonance. Blue labels are for resonances with Mimas, orange ones for resonances with Janus. Black dashed lines mark the position of constant pericentric and apocentric distances of particles, at the position of the inner and outer $\mathrm{G}$ ring edges, respectively. All particles inside this region are assumed to form part of the $\mathrm{G}$ ring.

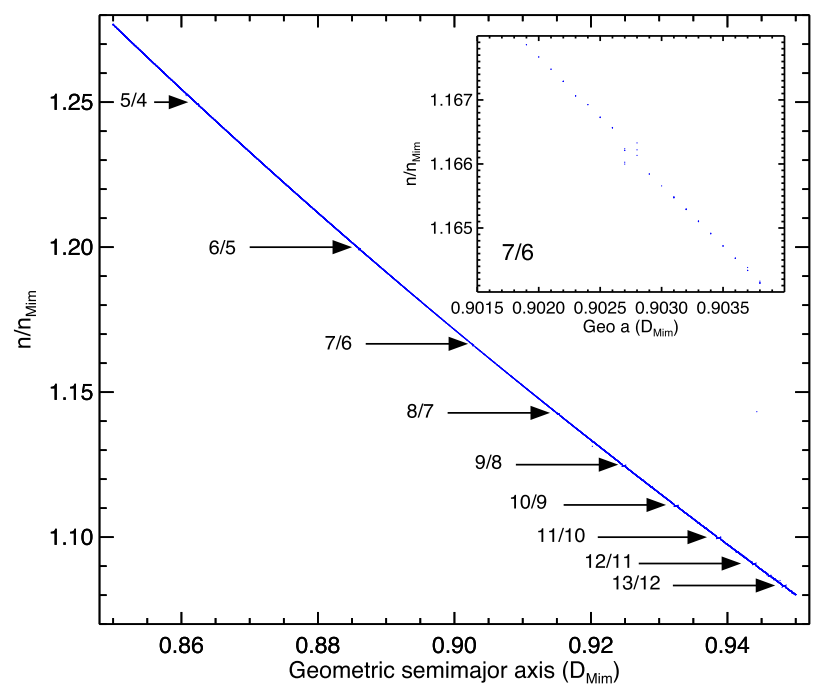

Figure 7. Frequency curve for the Aegaeon region. We plot the ratio of particle's mean motion, $n$, over Mimas mean motion, $n_{\text {Mim }}$, versus initial geometric semimajor axis of the particle. Plateaus in the curve correspond to the location of MMRs with Mimas. We highlight one of such plateaus for the 7:6 MMR close to the 7:6 CER, where Aegaeon librates to exemplify the flattening of the frequency curve. inhabiting such regions are expected to be small and therefore subject to drift forces like solar radiation pressure and plasma drag (see e.g. Sun et al. 2017, Madeira et al., in preparation).

Regarding the $\mathrm{G}$ ring, we know it is formed by $\mu \mathrm{m}$-sized particles and extends from $\sim 165000$ to $175000 \mathrm{~km}$ from Saturn centre, or from $\sim 0.8893$ to $0.9432 \mathrm{D}_{\text {Mim }}$ (Horányi et al. 2009). In Fig. 6, the location and size of the $\mathrm{G}$ ring are indicated by the double-headed black arrow. Also showed in the figure, in dashed lines, are the constant pericentre and apocentre of particles at the position of the inner and outer edges of the $\mathrm{G}$ ring, respectively. It is assumed that particles inside this region can form part of the ring, since particles outside of it (some of them with the same $a$ but larger $e$ ) would have pericentric and apocentric excursions farther away from the observed edges of the ring. Initially 3913 particles form part of the ring, while 2937 survive at the end of the simulation. That is, around 25 per cent of the $\mathrm{G}$ ring particles are lost due to collisions with Aegaeon. Besides, surviving particles have in average a $\log (D)=-4.3779971$, which implies a $t_{\text {Diff }}=1.079 \times 10^{7} \mathrm{yr}$. Such particles are dynamically heated, i.e. by increasing their inclination, from 0 to $0.00425^{\circ}$ in average, mainly due to the effect of MMRs with Mimas. This average inclination implies that the $\mathrm{G}$ ring is vertically widened in a short time period, by $\sim 12.6 \mathrm{~km}$, considering an average $a \approx 170000 \mathrm{~km}$ for the vertical excursions of particles $(a \times i)$. 


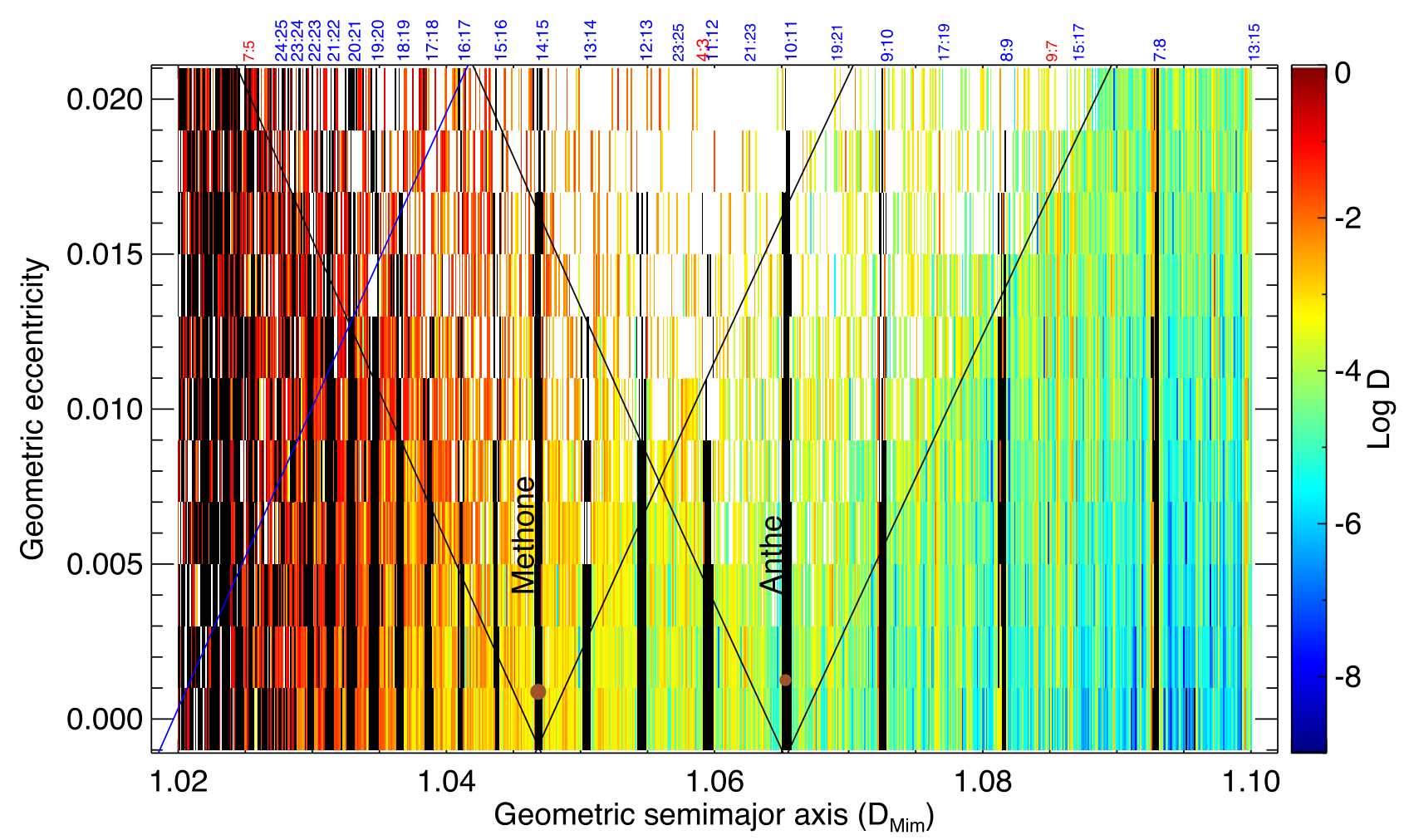

Figure 8. Diffusion map for the region around Methone and Anthe. The locations of the small moons are indicated by filled brown circles. Solid black lines stand for the apocentric and pericentric distances of particles at the position of pericentre and apocentre of the small moons, while the blue line is for the pericentric distance of particles at the apocentre of Mimas. As in Fig. 7, particles in first-order MMRs with Mimas are coloured black; closer to Mimas (to the left of the map) such resonances are tightly packaged. The resonant ratios for first- and second-order MMRs with Mimas (in blue) and Enceladus (in red) are shown at the top of the figure. An important percentage of the map surface is unstable due to the overlapping of collision regions of Mimas, Methone and Anthe.

\subsection{The region around Methone and Anthe}

For Methone and Anthe, we study the region around both moons in the same map due to their proximity. The simulation performed for this aim covers from 1.02 to $1.10 \mathrm{D}_{\mathrm{Mim}}$ in geometric $a$ and from 0 to 0.02 in geometric $e$. The steps in $a$ and $e$ are, as in the previous case, $1.0 \times 10^{-4}$ and $2 \times 10^{-3}$, respectively. This makes a total of 8800 test particles. Inclination is set to 0 as well as $\bar{\omega}, \Omega$ and $\lambda$. Particles are subject to the oblateness of Saturn, gravitational perturbations from the five large moons, plus Methone and Anthe, whose masses were considered to be $9.38 \times 10^{14} \mathrm{~g}$ for Methone and $3.5 \times 10^{14} \mathrm{~g}$ for Anthe. Regarding densities, we assumed values of $0.31 \mathrm{~g} \mathrm{~cm}^{-3}$ for Methone, following Thomas et al. (2013), while for Anthe we used a value of $0.35 \mathrm{~g} \mathrm{~cm}^{-3}$, by considering an average of the densities of the other three small moons.

The diffusion map for the region of Methone and Anthe is shown in Fig. 8. Brown filled circles mark the average position of the small moons. Black lines indicate the pericentric and apocentric distances of particles at the positions of the apocentres and pericentres of Methone and Anthe, respectively. The blue line marks the pericentric distance of particles at the apocentre of Mimas.

An important fraction of this map is unstable, mainly due to the overlapping of the collision regions of Mimas, Methone and Anthe. Nonetheless, some particles in the region interior to the orbit of Methone lie in MMRs with Mimas, which are tightly packaged the closer they are to the large moon. Another stability zone exists, located between the Methone and Anthe locations, while the predominantly stable region can be found beyond the orbit of Anthe, where the perturbative influence of Mimas is weaker. Inside the combined collision regions of Methone and Anthe, there are initially 4204 particles, out of which 2254 survive the whole simulation, therefore, around 46 per cent of the particles end up colliding with any of the moons. For the surviving particles an average $\log (D)=-2.9265259$ is found, which implies a $t_{\text {Diff }}=3.023 \times 10^{5} \mathrm{yr}$. This is a considerable shorter stability time than that found for the surviving particles inside the Aegaeon region; this since, although few, several of the Aegaeon surviving particles are those of smaller eccentricity, while others remain in resonance, avoiding larger perturbations from $\mathrm{Mi}$ mas and collision with Aegaeon. In this case, however, many particles that survive the simulation are part of the high eccentricity and largely perturbed region of the map, for which it is to be expected shorter diffusion time-scales.

Fig. 9 shows the frequency curve for the Methone/Anthe region. Interestingly, the unstable region close to Mimas is very easy to identify due to the scatter of the points (or $n / n_{\text {Mim }}$ values) away of the Keplerian trend. In the figure, we point out the existence of the Mimas MMRs: 7:8, 8:9, 9:10, 10:11, 11:12, 12:13, 13:14 and 14:15. The particles with the constant ratios responsible for the plateaus are coloured in black in the map of Fig. 8.

\subsection{The dynamical environment of Pallene}

The region around Pallene was studied through a simulation of test particles covering a patch of geometric $a$ versus $e$ phase space, going from 1.1 to $1.2 \mathrm{D}_{\mathrm{Mim}}$ in $a$, in steps of $1.0 \times 10^{-4}$. In $e$ the values 


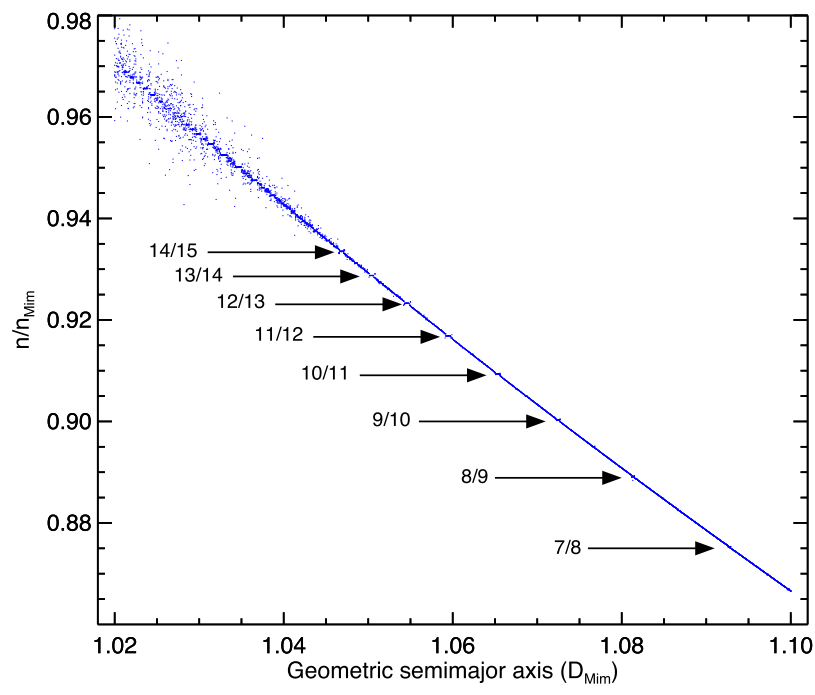

Figure 9. Frequency curve for the Methone and Anthe region. Same as in Fig. 7, we point to the plateaus of MMRs with Mimas. In this case, closer to Mimas (top-left in the figure) the unstable region is easily recognizable by the dispersion of the ratios.

run from 0.0 to 0.02 , in steps of $2 \times 10^{-3}$, totalizing 11000 test particles. They are subject to the gravitational perturbations from an oblate Saturn, the five large moons of the region plus Pallene. The mass of Pallene is taken to be $2.69 \times 10^{15} \mathrm{~g}$, with a density of $0.25 \mathrm{~g} \mathrm{~cm}^{-3}$ (Thomas et al. 2013).
The diffusion map for the Pallene region is shown in Fig. 10. Same as in previous cases, the average location of Pallene is mark by the filled brown circle. Solid black lines delimit the region where particles have the highest probability for colliding with Pallene. Dashed black lines delimit the apocentric and pericentric distances of particles at the position of the inner and outer edges of the Pallene ring, respectively. The width of the ring is accounted for with the double-headed arrow. The ring is about $2500 \mathrm{~km}$ in width and centred at the small moon (Hedman et al. 2009). Interestingly, the radial extend of the ring is constrained by the largest pericentric and apocentric excursions of Pallene itself.

We can see from Fig. 10 that Pallene does not lie close to any firstorder MMR, neither with Mimas or Enceladus, suggesting the nonresonant dynamics of the small moon and its ring. If by chance, the second-order MMRs 9:11 with Mimas and 13:11 with Enceladus lie close to the position of Pallene, these weak resonances seem unable to affect the dynamics of the small moon whatsoever. Nonetheless, a rich structure of resonances shapes this intermediate region between the major satellites Mimas and Enceladus, as can be seen from Fig. 2. In the map of Fig. 10, we plot in black the resonant particles in firstorder MMRs either with Mimas or Enceladus. At the top of the figure, we indicate the ratios of the resonances (in blue for Mimas, in red for Enceladus). It is clear that some of the vertical features visible on the map are not related to first-order MMRs; therefore, we also indicate, by labelling the ratio at the top of the figure, the location of second-order MMRs with Mimas and with Enceladus, with the same colour code. Despite the abundance of such first- and

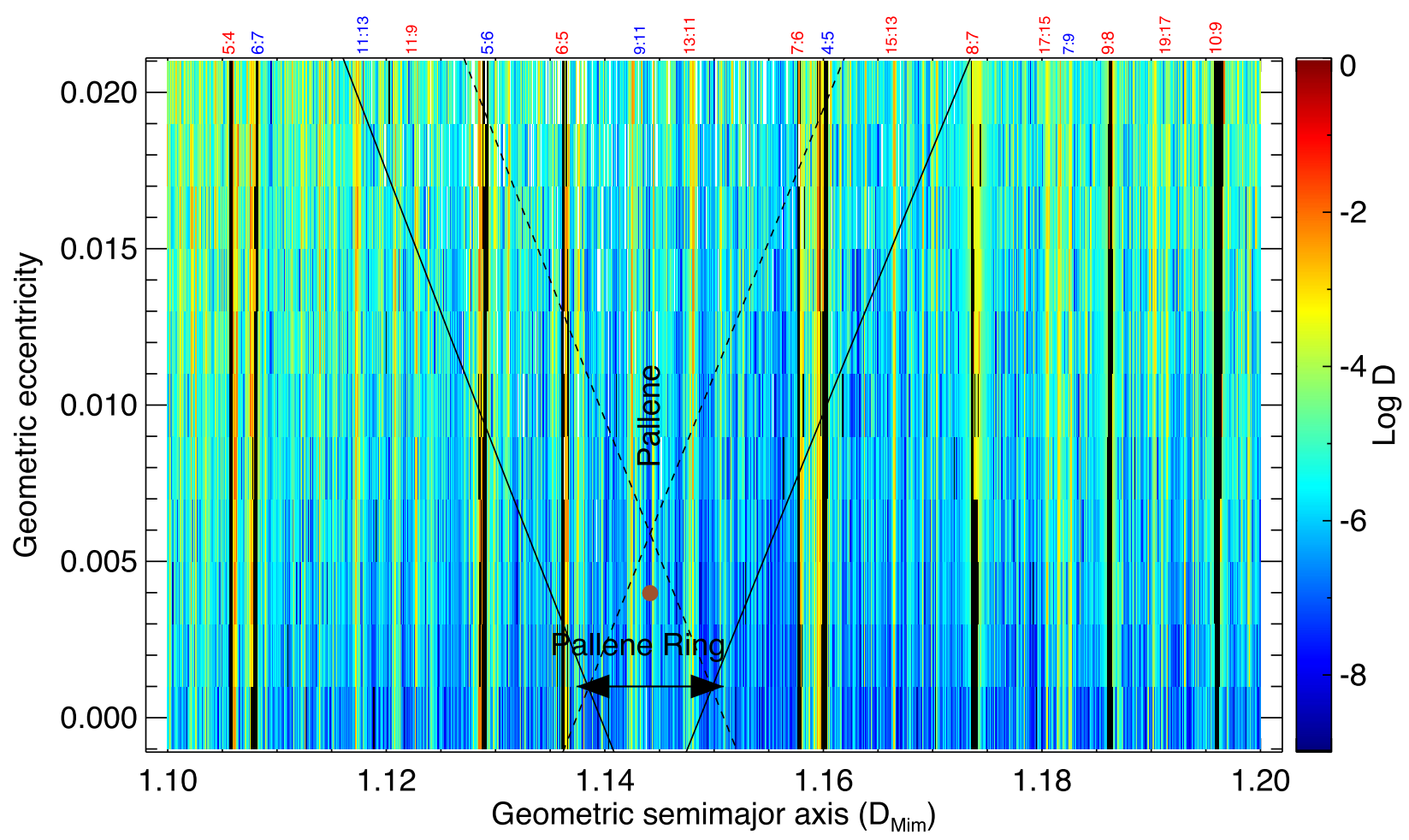

Figure 10. Diffusion map for the region around Pallene. The average location of Pallene is indicated by a filled brown circle. Solid black lines stand for the pericentric and apocentric distances of particles at the apocentre and pericentre distances of Pallene, respectively. Black dashed lines mark the position of constant pericentric and apocentric distances of particles, at the position of the inner and outer edges of the Pallene ring, respectively. The width and location of the ring is accounted by the double-headed arrow. All particles inside the region enclosed by dashed lines are assumed to form part of the ring. Particles in first order MMRs either with Mimas or Enceladus are coloured in black. The first and second order MMR ratios are shown at the top of the figure, in blue for resonances with Mimas and in red for resonances with Enceladus. Due to its small size and large $i$, Pallene is unable to clean up its orbit in only $\sim 18$ yr, or approximately 5700 of its orbital revolutions. 
second-order MMRs, all the region is globally very stable and only a minor fraction of particles are lost in our short time integration.

Inside the collision region of Pallene, there are initially 3522 particles, while 3375 of them survive at the end of the simulation. This is, less than $\sim 5$ per cent of the particles collide with Pallene in $\sim 18 \mathrm{yr}$. In average, for the surviving particles, we have a $\log (D)=-5.4219$, which implies a stability time of $t_{\text {Diff }}=8.365 \times 10^{7} \mathrm{yr}$, the longest for any of the regions studied so far. Regarding the Pallene ring, there are initially inside the region delimited by the dashed lines a total of 266 particles, out of which only one collided with Pallene. A $\log (D)=-6.0716074$ is found in average for the ring surviving particles, leading to $t_{\text {Diff }}=3.733 \times 10^{8} \mathrm{yr}$. The average inclination of the surviving ring particles is $0.001023^{\circ}$. This inclination implies vertical excursions of just $\sim 3.8 \mathrm{~km}$, considering an average $a \approx 212000 \mathrm{~km}$. This is an order of magnitude smaller than the vertical width of around $50 \mathrm{~km}$, determined by Hedman et al. (2009) for the Pallene ring. Nonetheless, we expect a larger value of the final average inclination in longer simulations. Such explorations are planned to be presented in a future work.

It is worth to note the high stability of the orbits inside the region of the maximum orbital excursions of Pallene. Even more, particles of small eccentricity lying close to the small moon, at least below $e \sim 0.006$, are those actually forming part of the ring and are expected to survive in a long-term basis. If the ring itself is a stable feature, it should then be formed by this low $e$ particles, leading in its way to a ring of small eccentricity also. Due to the limited number of Cassini images in which the Pallene ring is visible, it has not been possible to accurately determine the eccentricity of the ring, amongst other properties such as its mass. Nonetheless, the ring seems to follow closely the orbital path of the small moon. This fact implies that most likely the ring shares the same orbital properties of the parent moon. A further detailed study about the ring is planned to be presented in a future paper.

\section{LONG-TERM EVOLUTION OF AEGAEON, METHONE, ANTHE AND PALLENE}

In this section, we present the results of a single long-term simulation, lasting for $10^{5} \mathrm{yr}$, which included the nine large bodies of Table 1, and the oblate Saturn with physical parameters given in Table 2. In Fig. 11, we plot the evolution of the geometric orbital elements of the four small moons of Table 4. For them, we calculate the mean and maximum variations of the geometric parameters. Also, we apply the frequency analysis to the small moons in order to estimate a diffusion parameter and an associated diffusion timescale for them in the current configuration of the Saturnian system. Our results are summarize in Table 5.

Second and third columns of Table 5 show the mean and maximum variations of the geometric semimajor axis, $\bar{a}_{G e o}$, in units of $\mathrm{D}_{\mathrm{Mim}}$ (second column) and $\mathrm{km}$ (third column) for an easier interpretation. The largest variations in this parameter are those of Methone and Anthe. We can see that all the orbits of the small moons are highly stable over the $10^{5}-\mathrm{yr}$ simulation. The largest ones, for Methone and Anthe, are of only $\sim 28 \mathrm{~km}$. It has been suggested that those two moons are closer to a chaotic zone produced by the overlapping of the CER and LER resonances with Mimas (El Moutamid et al. 2014). The variations well above the $4 \mathrm{~km}$ separation of the CER and LER may lead to Methone and Anthe to be influenced by both resonances at different times; nonetheless, the combined perturbations are weak enough as being ineffective in leading to a chaotic evolution of their orbits in $10^{5} \mathrm{yr}$.

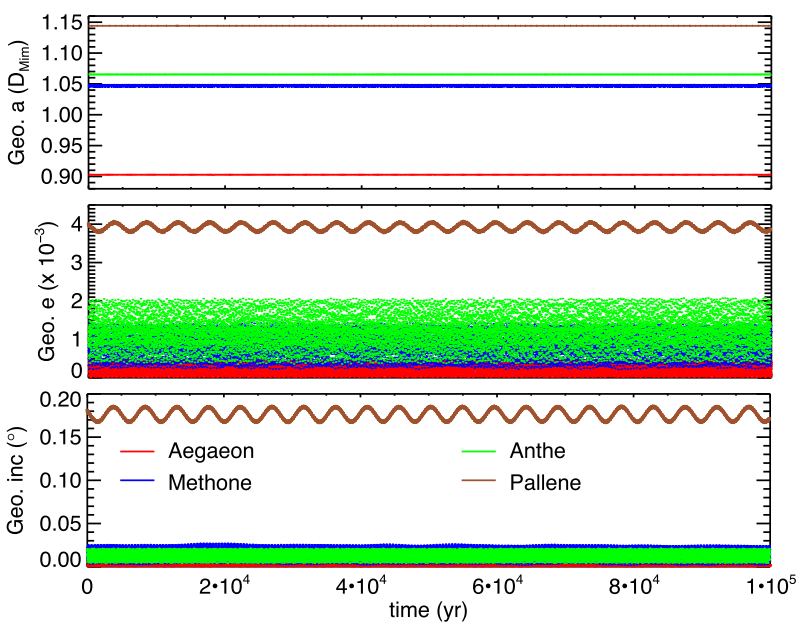

Figure 11. Long-term evolution of geometric orbital elements for Aegaeon, Methone, Anthe and Pallene. Variations in geometric semimajor axis (top panel) are hardly visible at this scale. Middle panel shows the evolution of geometric eccentricity, which larger variations are for Anthe (in green) and remarkably periodic for Pallene (brown). Bottom panel shows the evolution of geometric inclination. Pallene shows the larger variations, again periodic, besides the larger inclination amongst the small moons.

In the case of Aegaeon, the variations $<6.5 \mathrm{~km}$ implies that the moon is well trapped inside the 7:6 CER with Mimas. For Pallene, the variations of only $\sim 3 \mathrm{~km}$ turns it into the more stable of the small moons. Pallene is far from any first-order MMR, this allows it to avoid any strong perturbation from Mimas or Enceladus.

Regarding geometric $e$ and $i$, Anthe turns out to be the more perturbed in $\bar{e}_{\mathrm{Geo}}$, probably as a result of perturbations produced by the overlapping the CER and LER with Mimas, being that, as El Moutamid et al. (2014) have pointed out, Anthe is closer to the chaotic zone than any of the other small moons, although variations in this parameter for Methone are comparable. Regarding inclinations for Aegaeon, Methone and Anthe, they are also small, so the small moons remain very close to Saturn's equatorial plane; Aegaeon reaches maximum excursions above the plane of only $\sim 4.7 \mathrm{~km}$, while for Methone and Anthe vertical excursions are of $\sim 52$ and $\sim 46 \mathrm{~km}$, respectively. For Pallene, such excursions reach $\sim 653 \mathrm{~km}$.

The orbital behaviour of Pallene is interesting, showing that variations in $e$ and $i$ are periodic and clearly correlated. The period of such oscillations is $4762.21 \mathrm{yr}$ for both $e$ and $i$, and although its mean values of $e$ and $i$ are the largest amongst the small moons, it presents the more stable orbit. We can confirm the last statement by calculating the diffusion parameter and the associated diffusion time for the orbits of the small moons, as shown in the last two columns of Table 5. The value of $D$ is determined from the evolution of the same quantity $z^{\prime}(t)=a(t) \exp (i \lambda)$ as for the test particles of the diffusion maps. Here, $t_{\text {Diff }}$ gives us an estimation of the time required for an appreciable change in the orbital evolution of the small moons to be observed. As shown in the last column of Table 5, Pallene could remain stable for as long as $\sim 64 \mathrm{Myr}$ in its current orbit, while Aegaeon, Methone and Anthe could do the same for only $\sim 0.45$ Myr. This short-time period results from a crude estimation and could result from the large variations of the three small moons produced by the resonances they are trapped in. Nonetheless, for Pallene it seems to represent a confident value for the stability of the moon in a long-term basis. 
Table 5. Summary of mean values for the evolution of geometrical orbital elements and frequency analysis.

\begin{tabular}{|c|c|c|c|c|c|c|}
\hline Satellite & $\bar{a}_{\mathrm{Geo}}\left(\mathrm{D}_{\mathrm{Mim}}\right)$ & $\bar{a}_{\mathrm{Geo}}(\mathrm{km})$ & $\bar{e}_{\mathrm{Geo}} \times 10^{-3}$ & $\bar{i}_{\mathrm{Geo}} \times 10^{-2}\left(^{\circ}\right)$ & $\log (D)$ & $t_{\text {Diff }}(\mathrm{Myr})$ \\
\hline Methone & $1.0468417 \pm 1.531 \times 10^{-4}$ & $194229.97 \pm 28.40$ & $0.85436 \pm 0.61407$ & $1.5441 \pm 1.2678$ & -3.1172 & 0.473853 \\
\hline Pallene & $1.1441441 \pm 1.760 \times 10^{-5}$ & $212283.35 \pm 3.26$ & $3.9240 \pm 0.1677$ & $17.6289 \pm 1.0308$ & -5.3085 & 64.41534 \\
\hline
\end{tabular}

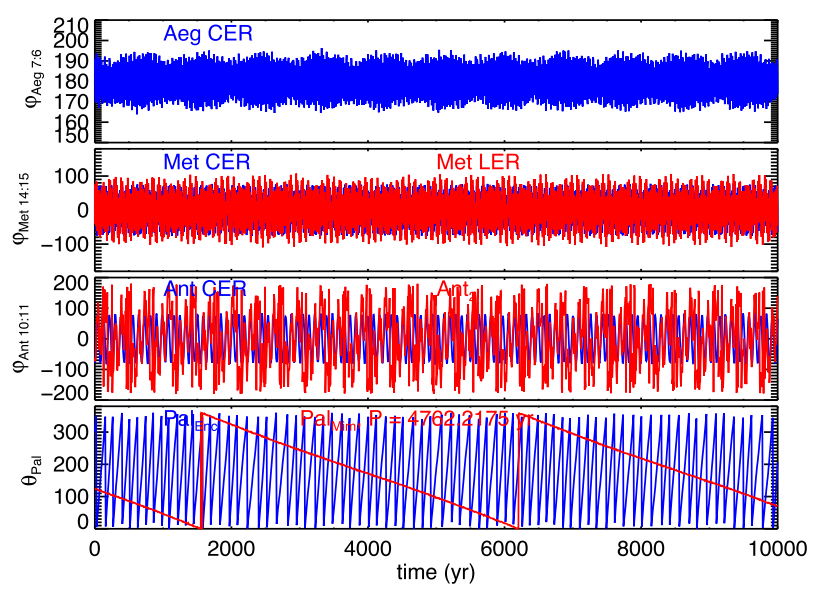

Figure 12. Long-term evolution of different resonant arguments of the four small moons. We show the same arguments as the ones presented in Fig. 1 but now for the initial $10000 \mathrm{yr}$ of the long-term simulation. All arguments for CER with Mimas (blue curves in three first panels) remain librating for Aegaeon, Methone and Anthe for the whole simulation time, so the moons remain in resonance. Pallene, on the other hand, is never in resonance. Nonetheless, the quasi-resonance with Mimas suggested by Callegari \& Yokoyama (2010) seems to perturb its orbit, inducing periodic variations in $e$ and $i$.

Regarding the resonant nature of the small moons, we show in Fig. 12 the long-term evolution of the same resonant arguments previously shown if Fig. 1, where we explored their short-term evolution. Now we show a period of $10000 \mathrm{yr}$ out of the $10^{5} \mathrm{yr}$ of our simulation, as the behaviour remains unchanged along the whole run. From Fig. 12, we can conclude that the three small moons currently trapped in CERs with Mimas will remain there for a long-term basis, as is shown by blue curves in the three first panels of Fig. 12. Such arguments remain librating for the whole time of the simulation. Aegaeon, Methone and Anthe are strongly affected by CER resonances and they could hardly escape from them, probably explaining the existence of their associated arcs. Indeed, if the small moons remain for a long time trapped in the resonances, the slow impact-ejecta processes that are believed to supply the arcs with dusty material could have plenty of time to act and originate such structures, even if the tiny grains are constantly removed due to the action of gravitational and non-gravitational forces, such as collisions, solar radiation and plasma drag.

For Methone, the argument associated with the LER with Mimas also remains librating for the whole simulation; therefore, this resonance also affects the dynamics of the small moon; this could explain the large variations in $a$ seen for Methone. In the case of Anthe, the argument $\varphi_{2}=11 \lambda_{\text {Ant }}-10 \lambda_{\text {Mim }}-\bar{\omega}_{\text {Ant }}-\Omega_{\text {Ant }}+\Omega_{\text {Mim }}$, shown in Section 3 , starts librating but then it changes and starts to circulate, then librates again repeating the cycle with a constant period and sequence. Although the erratic regime changes from libration to circulation are a signature of chaotic behaviour, in this case, the regularity in the behaviour of this argument seems not to lead to the chaotic evolution of Anthe.

In the case of Pallene, we show the two arguments previously presented in Section 3; now it is clear that both arguments circulate. The resonant argument associated with Mimas, $\theta=\bar{\omega}_{\text {Pal }}-\bar{\omega}_{\text {Mim }}-$ $\Omega_{\text {Mim }}+\Omega_{\text {Pal }}$, circulates with a period of $\sim 4762.21 \mathrm{yr}$; this is the same period as the one found for the long-term oscillations of $e$ and $i$. Such correlation implies that Mimas induces those variations on the orbit of the small moon, despite leaving it in a highly stable orbit.

\section{CONCLUSIONS}

In this work, we have explored the dynamical evolution of four Saturnian small moons: Aegaeon, Methone, Anthe and Pallene, as well as characterized their dynamical environment by using thousands of test particles surrounding them by means of short- and long-term simulations, going up to $10^{5} \mathrm{yr}$, that is, longer than any previous numerical exploration. We have considered the current configuration of the Saturnian system, including the oblateness of Saturn up to $J_{6}$ and the five largest moons close to the region of interest, namely Janus, Epimetheus, Mimas, Enceladus and Tethys. Through frequency analysis and the long-term evolution of the moons we have analysed their stability and that of the region surrounding them. The frequency analysis has allowed us to obtain a global dynamical perspective of the region inhabited by the small moons by using detailed but short time integrations. With it we gain an estimation of the stability times for particles in such regions and it also let us characterize the resonances involved in shaping or perturbing such regions. We have also found the following:

(i) All the four small moons are stable in a long-term basis. If the current configuration of the system would remain unchanged, that is, if the migration of the largest moons, mainly Mimas, is slow enough, then the stability time for the small moons would be at least $\sim 0.4 \mathrm{Myr}$ for Aegaeon, Methone and Anthe, and up to $64 \mathrm{Myr}$ for Pallene.

(ii) Aegaeon remains trapped in the 7:6 CER with Mimas, with maximum variations of only $\sim 6.4 \mathrm{~km}$ in $a$. Its $e$ remains small, and variations in $i$ implies maximum excursions above Saturn's equatorial plane of $4.7 \mathrm{~km}$.

(iii) Methone and Anthe share similar stability times, as well as variations of the same order in their orbital parameters. Methone librates in both the 14:15 CER and LER with Mimas, while Anthe remains in the 10:11 CER with Mimas but it is also close to the chaotic region produced by the overlapping of the CER and LER. The influence of two resonances on those small moons likely lead to largest variations than those observed for Aegaeon. For both Methone and Anthe, variations in $a$ are of $\sim 28 \mathrm{~km}$, while their $i$ imply vertical excursions of 52 and $46 \mathrm{~km}$, respectively.

(iv) Pallene is the most stable of the small moons analysed. It remains as non-resonant but, nonetheless, it suffers long-term perturbations from Mimas, though the quasi-resonance found by Callegari \& Yokoyama (2010). Such perturbations only affect the evolution 
of $e$ and $i$, inducing long-term oscillations, while its $a$ changes by only $\sim 3.2 \mathrm{~km}$.

(v) Regarding the $\mathrm{G}$ ring, 25 per cent of ring particles collide with Aegaeon, while the remaining are stirred in their $i$ up to $0.00425^{\circ}$ in average. This implies a vertical widening of $12.6 \mathrm{~km}$ in just $\sim 18 \mathrm{yr}$ of an initially flat distribution of particles.

(vi) The Pallene ring may be formed of particles of small eccentricity below $\sim 0.006$. Despite the larger size of Pallene compared to Aegaeon, Methone or Anthe, the small moon is unable to efficiently clean up its orbit, due to its anyway small size and large $i$. The average final inclination of ring particles is $0.001023^{\circ}$, which leads to a vertical width of $\sim 3.8 \mathrm{~km}$.

All the regions explored in this work have some stable zones, where no particles are found in the real system. If ever some particles existed in such regions, then some mechanism should be responsible for their removal. Such mechanisms are most likely the non-gravitational forces, such as solar radiation force and plasma drag, as it is expected that particles close to the small moons were originated when micrometeoroid IDPs hit the surfaces of the small moons, leaving ejecta that is also micrometrical in size.

The influences of non-gravitational forces have been studied for the regions of Aegaeon (for example in Madeira et al., in preparation), Methone and Anthe (Sun et al. 2017). The work exploring such shaping forces for the Pallene ring is under investigation.

\section{ACKNOWLEDGEMENTS}

We acknowledge an anonymous referee for insightful comments that help to improve this work. We thank the financial support from FAPESP (Proc. No 2016/01467-8 and 2011/08171-3). SGW also thanks CNPq project number 309254/2012-4. We acknowledge the use of the Saturn Cluster of the Grupo de Dinâmica Orbital e Planetologia at UNESP, campus of Guaratinguetá.

\section{REFERENCES}

Callegari N., Yokoyama T., 2010, in Fernandez J. A., Lazzaro D., Prialnik D., Schulz R., eds, Proc. IAU Symp. 263, Icy Bodies of the Solar System. Cambridge Univ. Press, Cambridge, p. 161

Chambers J. E., 1999, MNRAS, 304, 793
Cooper N. J., Murray C. D., Evans M. W., Beurle K., Jacobson R. A., Porco C. C., 2008, Icarus, 195, 765

Cooper N. J., Renner S., Murray C. D., Evans M. W., 2015, AJ, 149, 27

Correia A. C. M., Udry S., Mayor M., Laskar J., Naef D., Pepe F., Queloz D., Santos N. C., 2005, A\&A, 440, 751

El Moutamid M., Sicardy B., Renner S., 2014, Celest. Mech. Dyn. Astron., 118,235

Hedman M. M. et al., 2007, Science, 317, 653

Hedman M. M., Murray C. D., Cooper N. J., Tiscareno M. S., Beurle K., Evans M. W., Burns J. A., 2009, Icarus, 199, 378

Hedman M. M., Cooper N. J., Murray C. D., Beurle K., Evans M. W., Tiscareno M. S., Burns J. A., 2010, Icarus, 207, 433

Horányi M., Burns J. A., Hedman M. M., Jones G. H., Kempf S., 2009, Saturn from Cassini-Huygens. Springer, Netherlands

Jacobson R. A., Spitale J., Porco C. C., Owen W. M., Jr, 2006, AJ, 132, 711

Laskar J., 1990, Icarus, 88, 266

Laskar J., 1993, Celest. Mech. Dyn. Astron., 56, 191

Laskar J., Froeschlé C., Celletti A., 1992, Physica D: Nonlinear Phenomena, 56,253

Nadolski L., Laskar J., 2003, Phys. Rev. Spec. Top. Accelerators Beams, 6 , 114801

Nesvorný D., Morbidelli A., 1998, AJ, 116, 3029

Papaphilippou Y., 2014, Chaos, 24, 024412

Papaphilippou Y., Laskar J., 1998, A\&A, 329, 451

Porco C. C. et al., 2005, Science, 307, 1226

Renner S., Sicardy B., 2006, Celest. Mech. Dyn. Astron., 94, 237

Robutel P., Laskar J., 2001, Icarus, 152, 4

Šidlichovský M., Nesvorný D., 1996, Celest. Mech. Dyn. Astron., 65, 137

Spitale J. N., Jacobson R. A., Porco C. C., Owen W. M., Jr, 2006, AJ, 132, 692

Sun K.-L., Seiß M., Hedman M. M., Spahn F., 2017, Icarus, 284, 206

Thomas P. C., Burns J. A., Hedman M., Helfenstein P., Morrison S., Tiscareno M. S., Veverka J., 2013, Icarus, 226, 999

Valluri M., Merritt D., 1998, ApJ, 506, 686

This paper has been typeset from a $\mathrm{T}_{\mathrm{E}} \mathrm{X} / \mathrm{LAT}_{\mathrm{E}} \mathrm{X}$ file prepared by the author. 